\title{
BEITRÄGE
}

ZUR

\section{VERGLEICHENDEN OSTEOLOGIE}

DES

\section{SCHAFES UND DER ZIEGE.}

\section{INAUGURAL-DISSERTATION \\ DER}

HOHEN PHILOSOPHISCHEN FACULTÄT DER

UNIVERSITÄT I.EIPZIG

ZUR

ERLANGUNG DER DOKTORWÜRDE VORGELEGT VON

\section{CARL BÜTZLER.}

MIT FÜNF LICHTDRUCK-TAFELN.

\subsection{5}

LEIPZIG

DRUCK VON C. G. NAUMANN 1896. 

Die Osteologie bildet einen Theil der deskriptiven Zoologie und beschreibt die Knochen der Thiere als solche in einem von allen Weichgebilden getrennten Zustande. Die vergleichende Osteologie der Haussäugethiere finden wir von den Veterinär-Anatomen ziemlich weit ausgebildet und in den Handbüchern von Gurlt ( $\left.{ }^{1}\right)$, Ellenberger und Müller $\left({ }^{2}\right)$ bezw. Leisering und Müller, sowie Franck $\left({ }^{3}\right)$ und Sussdorf $\left({ }^{14}\right)$ ausführlich dargelegt. Jedoch sind in diesen Werken unter den Haussäugethieren hauptsächlich Pferd und Rind berücksichtigt; auch Hund, Katze und Schwein werden noch ziemlich eingehend abgehandelt, Schaf und Ziege dagegen nur als kleine Wiederkäuer zusammengefasst, in den Gegensatz zum Rinde gestellt und gemeinsam in Kürze erwähnt. Der Grund hierfür dürfte besonders in dem Umstande zu suchen sein, dass die beiden letzteren Thierarten im Verhältniss zn den grossen Hausthieren für das Studium der Thierheilkunde wenig Interesse bieten.

Ueber Schafzucht haben H. von Nathusius $\left({ }^{4}\right)$, Settegast $\left({ }^{5}\right)$, Rhode $\left({ }^{6}\right)$, Mentzel ( $\left.{ }^{7}\right)$ u. A. geschrieben; unter diesen ist $H$. von $\mathrm{N}$ athusius besonders hervorzuheben, der im zweiten Theile seines, als Vorträge über Rassenkenntniss und Viehzucht betitelten Werkes die Osteologie des Schafes unter Berücksichtigung aller bekannten Rassen eingehend abgehandelt hat. Vor einigen Jahren veröffentlichte Prof. Paul Martin (8) in Zürich in der von Prof. Dr. Ostertag herausgegebenen Zeitschrift für Fleisch- und Milchhygiene eine für die praktische Fleischschau äusserst werthvolle Arbeit unter dem Titel „Die Beurtheilung der Herkunft des Fleisches nach den vorhandenen Knochentheilen"; Martin ( $\left.{ }^{8}\right)$ vergleicht zu diesem Zwecke die Knochen von Pferd - Rind, Schaf - Ziege Reh, Hund - Schwein, sowie Hase - Kaninchen - Katze - Hund mit einander und gelangt dabei zu beachtenswerthen Resultaten.

Für die Zwecke einer eingehenden vergleichenden Osteologie des Schafes und der Ziege ist es durchaus erforderlich, die Rasseneigenthümlichkeiten einer jeden von diesen beiden Thierarten, namentlich des Schafes, so viel als möglich zu berücksichtigen; denn erst nach Ausschluss aller bei derselben Thierspezies in Frage kommenden Verschiedenheiten sind wir in der Lage, zwischen Schaf und Ziege feststehende Unterschiede aufzufinden, welche geeignet sind als sichere Unterscheidungsmerkmale $\mathrm{zu}$ dienen und einen Beitrag $\mathrm{zu}$ der vergleichenden Osteologie des Schafes und der Ziege zu liefern. Ausserdem ist es nothwendig, auf die Entwicklungsgeschichte beider Thierarten 
zurückzugreifen, um zu ermitteln, in wie weit die an den ausgewachsenen Thieren aufgefundenen Unterschiede bereits an den Embryonal- bezw. Jugendzuständen derselben vorhanden und für die weitere Entwicklung mit bestimmend sind.

Nachdem ich schon seit längerer Zeit während meiner Thätigkeit als Betriebsleiter eines öffentlichen Schlachthofes umfangreiche Vorstudien für die vorliegende Abhandlung gemacht hatte, führte ich die genaueren Untersuchungen und Messungen im zoologischen Institut der Universität zu Berlin unter Leitung des Geh. Regierungs-Rathes Herrn Prof. Dr. Schulze aus; auf dessen Empfehlung hin hatte Herr Prof. Dr. Nehring an der landwirthschaftlichen Hochschule zu Berlin die Freundlichkeit, mir für meine Untersuchungen die Benutzung der $\mathrm{N}$ ath usius'schen Hundisburger Sammlung zu gestatten, welche bei der Gründung der landwirthschaftlichen Hochschule für das zoologische Institut käuflich erworben wurde. Für das rege Interesse, welches beide Herren sowie Herr Geheimrath Prof. Dr. Leuckart in Leipzig meiner Arbeit entgegenbrachten, erlaube ich mir, denselben auch an dieser Stelle meinen Dank auszusprechen.

Aus der reichhaltigen Nathusius'schen Sammlung wählte ich für meine Untersuchungen zwei hinsichtlich der Grösse beträchtlich von einander differirende Schafrassenskelete aus, und gleichfalls zwei verschiedene Ziegenskelete; dieselben habe ich nun eingehend untersucht und mit einander verglichen, ohne jedoch die zahlreichen übrigen Skelete von Schafen und Ziegen, welche in der Sammlung enthalten sind, ausser Acht zu lassen. Die Vergleichung dieser nachstehend aufgeführten Skelete hat mit Hülfe von exakt ausgeführten Messungen manche neue Resultate ergeben, welche ich in vorliegender Abhandlung darlegen möchte.

Das Inventarverzeichniss der Hundisburger Sammlung enthält über die von mir ausgewählten. Skelete folgende Bemerkungen:

Nr. I 558. Ovis aries + , graue, kurzschwänzige Tieflandsrasse von der Ems. Vollständiges Skelet, durch Israel in Weener (Ostfriesland) erhalten und im Winter $187 \mathrm{I} / 72$ in der Berliner Thierarzneischule präparirt.

vert. coll. 7

dors. I 3

lumb. 7 !

sac. 3 !

caud. I6!

Nr. I 595. Ovis aries $q$, Heidschnucke. Vollständiges Skelet. Im August I 872 durch Herrn Focke in Ebstorf aus Wulfsrode, mitten in der Lüneburger Heide gelegen, von $H$. Refordt gekauft, "einer der wenigen, der noch einige ganz reine Heidschnucken besitzt". Möglichst normal gebautes Thier.

vert. coll. 7

dors. I 3 , cost. I 3

lumb. 6

cruc. 3

caud. I 5 incl. letztes Rudiment. 
Griffelbein am linken Vorderfuss vorhanden, rechts wahrscheinlich beim Präpariren verloren.

Nr. 1556. Capra hircus L. + , gehörnt. Schwarze Ziege vom Schafmeister Bertram in Hundisbnrg gezogen und als Milchziege gehalten, ungefähr 8 Jahre alt. Vollständiges Skelet, präparirt in der Thierarzneischule zu Berlin im Winter 1871/72.

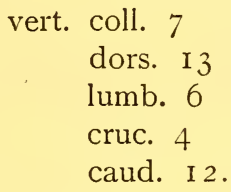

Nr. 1568. Capra hircus L. q, gehörnt. In Berlin 187 I geschlachtet und in der Thierarzneischule vom Anatomie-Wärter Knip präparirt.

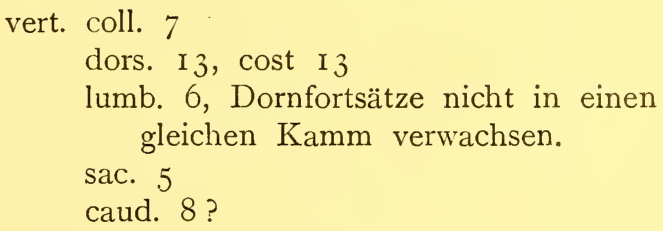

Der letzte Schwanzwirbel war beim lebenden Thier durch Schnitt oder Biss verletzt, aber vernarbt.

A n m. $23 \mathrm{~mm}$ langes Griffelbein zum ersten $\mathrm{Mal}$ an einer Ziege gesehen! fehlt also nicht!

Während von den vorliegenden Skeleten die der beiden Schafe, namentlich in Bezug auf die Grössenverhältnisse erheblich differiren, ist dies bei den Ziegenskeleten nur in geringem Maasse der Fall. Im Folgenden habe ich daher bei der Beschreibung der einzelnen Ziegenknochen die zwischen den Skeletten Nr. I556 und I568 etwa bestehenden Verschiedenheiten unmittelbar aufgeführt, im Uebrigen aber, um Wiederholungen $\mathrm{zu}$ vermeiden, beide Ziegenskelete gemeinsam abgehandelt.

Hinsichtlich der Eintheilung des Stoffes bin ich dem Beispiele der Veterinär-Anatomen gefolgt. Ich beginne demnach mit der Beschreibung und Vergleichung der Wirbelsäule beider Thierarten, da sie die Grundlage des Rumpfes und somit des ganzen Skelets bildet; an dieselbe fügen sich die Knochen an, welche mit $\mathrm{zu}$ denen des Rumpfes gerechnet werden, nämlich Rippen, Brustbein und die beiden Beckenbeine. Alsdann nach der Betrachtung des Kopfes habe ich die Beschreibung der in Betracht kommenden Knochen der vorderen und schliesslich die der hinteren Extremitäten folgen lassen.

\section{A. Halswirbel.}

Bei den verschiedenen Schafrassen ist der erste Halswirbel von verschiedener Grösse, jedoch von ziemlich gleicher Form, was schon durch den stets parallelen Verlauf der die Gestalt des Atlas wesentlich 
bestimmenden Flügelränder bedingt wird. Der grösste Querdurchmesser fällt nach $\mathrm{H}$. von $\mathrm{Nathusius} \mathrm{in} \mathrm{der} \mathrm{Regel} \mathrm{in} \mathrm{die} \mathrm{Achse} \mathrm{der} \mathrm{vorderen}$ Flügellöcher. Bei den beiden Schafrassen, von welchen die vorliegenden Skelette stammen, sind die Grössenverhältnisse des Atlas folgende:

Marschschaf (ostfriesisches):

Querdurchmesser:

Längsdurchmesser:

$83 \mathrm{~mm}$

$3 \mathrm{I} \mathrm{mm}$

Längsdurchmesser der Flügel: $63 \mathrm{~mm}$.

Heidschnucke:

Querdurchmesser:

Längsdurchmesser:

$56 \mathrm{~mm}$

Längsdurchmesser der Flügel: $40 \mathrm{~mm}$.

Der erstere Längsdurchmesser wird durch Messung der Längsachse des Arcus anterior gefunden. H. von Nathusius (4) stellt auf diese Weise bei den kurzen, gedrungenen Wirbeln ein Verhältniss von $I: 4$, bei den langgestreckten ein solches von $\mathrm{I}: 2,5$ fest.

Die beiden ersten Halswirbel des Marschschafes und der Heidschnucke mit einander verglichen, lassen erkennen, dass die Ausschnitte zwischen den beiden Gelenkpfannen am vorderen Ende von verschiedener Tiefe sind; während dieser Ausschnitt an dem Atlas der Heidschnucke mehr rundlich erscheint, ist er bei dem kurzschwänzigen Tieflandsschafe breiter und nahezu viereckig. Bei letzterer Schafrasse finde ich die Flügel des Atlas durch starke Fortsätze nach hinten verlängert und weit über den Rand der hinteren Gelenkfläche hervorragend, bei der Heidschnucke dagegen sind die Flügelfortsätze verhältnissmässig schwach entwickelt. Auch der hintere Ausschnitt des Arcus posterior erscheint bei dem Marschschafe ziemlich viereckig, bei der Heidschnucke mehr rundlich gestaltet. Die kammartige Hervorragung an der unteren Fläche des vorderen Wirbelbogens, das Tuberculum anterius, bildet beim Schafe eine rauhe, scharf begrenzte Erhabenheit mit rundlicher Basis und liegt bei den vorliegenden Schafskeleten auf der Mitte des Arcus anterior. Ebenso zeigt sich bei dem Marschschafe und bei der Heidschnucke das Tuberculum posterius gleich stark entwickelt und stellt eine rauhe, aus mehreren kleinen Knochenleisten zusammengesetzte Erhabenheit dar. Am Atlas des Schafes findet sich nur ein Flügelloch jederseits und zwar übereinstimmend bei allen Rassen, deren Skelete im zoologischen Museum vorhanden sind; die Oeffnung ist meistens ziemlich gross, besitzt eine längliche Form und dient dem ersten Halsnerven sowie der Hinterhauptsarterie zum Durchtritt. $\mathrm{H}$. von Nathusius $\left({ }^{4}\right)$ giebt an, dass sich aussen über das Foramen alae zuweilen eine Knochenbrücke hinzieht und dasselbe in zwei Theile theilt, wodurch scheinbar zwei Löcher neben einander liegen; an dem Atlas des ostfriesischen Marschschafes ist eine solche Knochenbrücke durch einen kleinen, zahnförmigen, in das Flügelloch hineinragenden Fortsatz angedeutet.

An dem ersten Halswirbel der Ziege konnte ich durch Vergleichung mit demselben Wirbel des Schafes folgende Unterschiede feststellen. 
Der Arcus anterior ist bei der Ziege schmäler im Verhältniss zur Länge des Atlas, was auch aus nachstehenden Maassen hervorgeht:

\section{Querdurchmesser: $\quad 70 \mathrm{~mm}$}

Längsdurchmesser des Arc. ant.: $30 \mathrm{~mm}$

Längsdurchmesser der Flügel: $6 \mathrm{I} \mathrm{mm}$.

Nach H. von Nathusius $\left({ }^{4}\right)$ ist bei den Ziegen das Verhältniss der Dimensionen der Längsachse des vorderen Bogens und des grössten Querdurchmessers der Flügel des Atlas ebenfalls variabel; jedoch sind nach seiner Angabe gleich kurze Wirbel wie bei den kurzhalsigen Schafen noch nicht beobachtet worden. Bei der Ziege ist das Tuberculum anterius höher und schmäler, also schärfer ausgeprägt und liegt nicht in der Mitte wie beim Schafe, sondern im hinteren Drittel des Wirbelbogens; es setzt sich nach vorn bedeutend schwächer werdend fort und ragt in den Ausschnitt zwischen den beiden Gelenkfortsätzen hinein, wo es als ein ziemlicn kräftiger, zahnförmiger Vorsprung diesen vorderen Ausschnitt, abweichend vom Schafe, in zwei scharf von einander getrennte, halbmondförmige Ausschnitte theilt. (Tafel I, Figur I.) Bei dem Ziegenskelet Nr. I568 ist dieser zahnförmige Fortsatz verhältnissmässig noch stärker entwickelt und bildet sogar zwei Spitzen. Bei der Ziege sind ferner die Flügelfortsätze nahezu um die Hälfte länger als beim Schafe (Tafel I, Figur I-3.) Dazu kommt noch, dass die vorderen und hinteren Ausschnitte des Arcus posterior bei der Ziege bei weitem seichter und schmäler, aber niemals viereckig sind wie beim Schafe.

Auch beim Epistropheus (Tafel I, Figur 5 und 6) finden wir bei den einzelnen Schafrassen eine verschiedene Länge, was bei den von mir zum Vergleiche gewählten Schafskeleten besonders auffällt. Die Verschiedenheit der Höhe und Stärke findet sich an dem Kamm des zweiten Halswirbels am deutlichsten ausgeprägt, jedoch zeigt derselbe noch bei dem Marschschafe und bei der Heidschnucke eine verschiedene Gestalt: Bei dem erstgenannten Thiere ist der Kamm der Axis vorn abgerundet und hinten scharfkantig, eine ziemlich weit nach rückwärts ragende, breite rauhe Beule bildend; bei der Heidschnucke dagegen zeigt sich der Kamm vorn spitz und vorspringend, hinten aber rundlich und ziemlich rasch abfallend. Dadurch werden vorn und hinten zwischen Kamm und Wirbelbogen zwei ganz verschiedenartige Ausschnitte gebildet. H. von Nathusius giebt an, dass durch die starke Entwicklung des Kammes bei den kurzhalsigen Schafen der ganze Wirbel höher als in irgend einer Dimension breit oder lang ist. Nach Franck ( ${ }^{3}$ ) fehlt das Querfortsatzloch sowie das hintere Zwischenwirbelloch mitunter beim Schafe, was $H$. von Nathusius $\left({ }^{4}\right)$ mit Recht bestreitet; auch an dem zweiten Halswirbel der vorliegenden Schafskelete sind diese Foramina vorhanden.

Im Gegensatz zu dem Epistropheus des Schafes ist derselbe Wirbel bei der Ziege viel schmäler und schlanker. Der cylindrische Zahnfortsatz besitzt bei der Ziege meist noch eine besonders abgesetzte, kugelförmige Hervorragung, niemals aber zeigt er sich an seinem vorderen Rande ausgebuchtet, wie dies bei den beiden Schafrassen der Fall ist. 
(Tafel I, Figur 4-6.) Der Kamm auf dem Wirbelbogen ist an beiden Ziegenskeleten um ein Drittel länger als der des Schafes und ragt mit seinem vorderen und hinteren Ende bedeutend über den Wirbelkörper hinaus; er bildet in Folge dessen zu beiden Enden des Wirbelkörpers bei der Ziege viel schärfere Ausschnitte als dies beim Schafe der Fall ist. Während am 3., 4., 5. und 6. Halswirbel der Ziege gleichwie beim Schafe ein Querfortsatzloch vorhanden ist, fehlt ein solches am Epistropheus der Ziege; auch an allen übrigen Ziegenskeleten der zoologischen Sammlung fehlt das Querfortsatzloch, dagegen an keinem einzigen von den zahlreichen Schafskeleten. Das Zwischenwirbelloch ist dafür bei der Ziege etwa doppelt so gross als beim Schafe.

Am 3., 4. und 5. Halswirbel des Schafes hat der obere Bogen die Form eines länglichen Vierecks, bei welchem die vorderen und hinteren schiefen Fortsätze die Ecken bilden. Bei den beiden Schafrassen habe ich folgende Maasse gefunden und dieselben in der Mitte der Wirbelkörper an der ventralen Fläche aufgenommen:

$\begin{array}{rcccc}\text { Marschschaf: } & 3 \cdot & 4 . & 5 . & \\ \text { Länge: } & 45 & 42 & 39,5 & \mathrm{~mm} \\ \text { Breite: } & 29 & 34 & 35 & \mathrm{~mm} \\ \text { Heidschnucke: } & & & & \\ \text { Länge: } & 3 \mathrm{I} & 29 & 25 & \mathrm{~mm} \\ \text { Breite: } & 2 \mathrm{I} & 25 & 28 & \mathrm{~mm}\end{array}$

Nach H. von Nathusius ( $\left.{ }^{4}\right)$ ist hier bei den einzelnen Individuen derselben Rasse die Geschlechtsdifferenz als ausgeglichen $\mathrm{zu}$ betrachten; es ist demnach bei dem einzelnen Individuum die vom 3. bis 5. Wirbel gebildete Halspartie absolut kürzer als bei dem anderen Individuum derselben Rasse. Gräte und Kamm auf dem oberen Bogen dieser drei Halswirbel bieten noch grössere Verschiedenheiten dar als beim Epistropheus. Bei dem kurzschwänzigen Tieflandsschafe besitzt die Gräte des 3. Halswirbels in der Mitte eine deutliche, längsverlaufende Rinne und bildet so gleichsam einen Doppelkamm. Diese Rinne zeigt sich auch auf dem 4. Halswirbel noch angedeutet; der 5. Wirbel dagegen trägt einen ziemlich starken Dornfortsatz. Anders verhalten sich diese Halswirbel bei der Heidschnucke; hier sind ausschliesslich Dornfortsätze vorhanden, welche allerdings ziemlich schwach entwickelt, vom 3. bis zum 5. Wirbel allmählich etwas an Höhe und Stärke zunehmen.

Bei der Ziege besitzt der 3., 4. und 5. Halswirbel je einen Dornfortsatz; diese Fortsätze sind schlank und kräftig und fehlen bei keinem der im Museum vorhandenen Ziegenskelete. Der Dornfortsatz des 5. Halswirbels ist im Gegensatz zu denen der beiden vorhergehenden stark nach vorn gerichtet. Müller $\left({ }^{2}\right)$ giebt an, dass die Halswirbel des Schafes und der Ziege im Verhältniss zur Körpergrösse etwas länger sind als die des Rindes. Nach meinen Beobachtungen trifft dies hauptsächlich für den 3., 4. und 5. Halswirbel zu, da dieselben allein die erheblichere Länge des Halses bei den kleinen Wiederkäuern bedingen, was auch $H$. von Nathusius ( ${ }^{4}$ ) hervorhebt. 
Der 6. und 7. Halswirbel sind beim Schafe im Verhältniss zu den übrigen Halswirbeln kurz und unterscheiden sich namentlich durch die Länge ihrer Dornfortsätze von einander. Nach H. von Nathusius ( ${ }^{4}$ ) ist der Dorn des 7. zuweilen nicht höher als der des 6. Halswirbels, zuweilen aber auch doppelt so hoch als jener. Besondere Unterschiede zeigen noch die vorliegenden Skelete: der Dornfortsatz des 6. Halswirbels ist sowohl beim Marschschafe als auch bei der Heidschnucke nach vorn gerichtet und hat bei beiden die gleiche Höhe. Beim Marschschafe geht jedoch der breite Dornfortsatz in eine Fläche aus, an welcher wir zwei, durch einen mittleren und zwei seitliche rauhe Kämme gebildete, rinnenförmige Vertiefungen unterscheiden können; bei der Heidschnucke dagegen ist der Dornfortsatz desselben Wirbels dreieckig und endet mit einer etwas abgestutzten Spitze. Die Maasse des 7. Halswirbels sind folgende:

Marschschaf:

Breite des Wirbelkörpers: $35 \mathrm{~mm}$

Höhe des Dornfortsatzes: $24 \mathrm{~mm}$

Heidschnucke:

Breite des Wirbelkörpers: $18 \mathrm{~mm}$

Höhe des Dornfortsatzes: $30 \mathrm{~mm}$.

Bei der Heidschnucke ist der Dornfortsatz des 7. Halswirbels nach hinten gerichtet und hat eine konische, seitlich etwas zusammengedrückte Gestalt; er gleicht in seiner Form dem Hakenzahn eines Ebers. Im Gegensatze hierzu ist der Dornfortsatz des letzten Halswirbels beim Marschschafe nach vorn gerichtet und erscheint bedeutend kürzer, obgleich der Wirbelkörper selbst nahezu die doppelte Breite besitzt. Der Winkel, welcher von dem Dornfortsatze des 7. Halswirbels und dem des I. Rückenwirbels gebildet wird, ist wie im vorliegenden Falle, nach H. von Nathusius $\left(^{4}\right)$ überhaupt bei den verschiedenen Schafrassen von verschiedener Grösse.

Bei der Ziege sind ebenso wie an den drei mittleren Halswirbeln die Dornfortsätze an den beiden letzten Halswirbeln bedeutend länger, spitzer und scharfkantiger als beim Schafe. Der Dornfortsatz des 7. Halswirbels ist an den vorliegenden Ziegenskeleten sogar nahezu um das Doppelte länger als bei dem Marschschafe; während er bei letzterem nur $25 \mathrm{~mm}$ misst, beträgt seine Länge bei der Ziege über $46 \mathrm{~mm}$. Ferner finden wir sämmtliche Dornfortsätze bei der Ziege mehr schief nach vorn umgelegt, was auch Martin $\left(^{8}\right)$ besonders hervorhebt. Bei der Ziege sind ausserdem die Querfortsätze der Wirbel schwächer und stehen nicht so weit ab vom Wirbelkörper als beim Schafe; dieselben sind dünner und zierlicher und besitzen schärfere Seitenränder. Schliesslich zeigt die ventrale Fläche des Körpers des 2. bis 5. Halswirbels eine viel schärfer ausgeprägte Leiste, als beim Schafe.

\section{B. Rückenwirbel.}

Die Zahl der Rückenwirbel beträgt, wie auch an den vorliegenden Schafskeleten, in der Regel I3, mitunter auch I2; jedoch sind nach Müller ( ${ }^{2}$ ) und Nathusius ( $\left(^{4}\right)$ I 2 Rückenwirbel oder gar I4 als eine 
seltene Ausnahme zu betrachten. H. von Nathusius fügt p. 90 hinzu: „Von Rassedifferenzen kann in dieser Beziehung keine Rede sein."

Bei dem Marschschafe und der Heidschnucke ist die Länge der Rückenwirbel folgende, wenn wir die Abstände zwischen den Rändern des Gelenkkopfes und den Gelenkpfannen am ventralen Theile der Wirbelkörper messen:

Marschschaf:

\begin{tabular}{|c|c|c|c|}
\hline I. & $2 .-9$ & $\begin{array}{c}\text { IO. I } 2 . \\
27-29\end{array}$ & $\begin{array}{l}\mathrm{I} 3 . \\
3 \mathrm{I} \mathrm{mm}\end{array}$ \\
\hline & \multicolumn{3}{|c|}{ Heidschnucke: } \\
\hline & $\begin{array}{c}2 .-9 . \\
\text { I } 7,5\end{array}$ & IO. - I 2. & $\begin{array}{l}\text { I } 3 . \\
22\end{array}$ \\
\hline
\end{tabular}

Bei beiden Schafrassen ist demnach der I. der kürzeste, der I 3 . der längste unter den Rückenwirbeln; vom 2.-9. ist die Länge ziemlich gleich, der IO., I I. und I 2. Wirbel sind jeder ein wenig länger als der vorhergehende. Analog diesen Längenverhältnissen wechseln auch die Breitenmaasse der einzelnen Rückenwirbel. Wenn wir den Abstand der lateralen Ränder der Querfortsatzpfannen messen, welche die Gelenkflächen der Rippenhöcker aufnehmen, so ergiebt sich, dass der erste und der letzte die breitesten von sämmtlichen Wirbeln sind. Ich fand für die Breite folgende Zahlen:

\begin{tabular}{cccc}
\multicolumn{4}{c}{ Marschschaf: } \\
I. & $2 .-8$. & $9 .-\mathrm{I} 2$. & I 3. \\
60 & $53-45$ & $5 \mathrm{O}$ & $63 \mathrm{~mm} ;$ \\
\multicolumn{4}{c}{ Heidschnucke: } \\
I. & $2 .-8$. & $9 .-\mathrm{I} 2$. & I 3. \\
4 I & 35 & $37-35$ & 4 I mm.
\end{tabular}

Die Rückenwirbel des Schafes sowie die der Ziege, was schon vorweg bemerkt werden soll, besitzen im Gegensatz zu denen des Rindes keine Löcher im Bogen. Nach Müller $\left(^{2}\right)$ ist der Kamm an der ventralen Fläche des Körpers nur an den beiden letzten Rückenwirbeln angedeutet; bei der Heidschnucke finde ich dies bestätigt, bei dem ostfriesischen Marschschafe dagegen lässt sich bereits vom 8. Wirbel ab ein ziemlich scharf ausgeprägter Kamm nachweisen, während vom 2.-7. Rückenwirbel an dieser Stelle eine leistenförmige Erhabenheit zu erkennen ist.

Die Dornfortsätze der Rückenwirbel stehen beim Schafe hinsichtlich ihrer Breite im Verhältniss zur Länge der Wirbelkörper; in Bezug auf Höhe und Richtung bieten dieselben bei dem Marschschafe und bei der Heidschnucke grosse Verschiedenheiten dar. Bei dem erstgenannten Thiere hat der Dornfortsatz des I. Rückenwirbels die gleiche Höhe wie der 9., der 3. ist der längste; vom 4. an nimmt die Höhe bis zum letzten allmählich ab: der letzte ist also der kleinste, zugleich aber auch der breiteste. Nach $\mathrm{H}$. von Nathusius $\left({ }^{4}\right)$ ist jedoch das Verhäitniss der Höhe nicht proportional zur Nummer des Wirbels, ebensowenig wie der Grad der Neigung des Dornfortsatzes zur Nummer des 
selben Wirbels. Die Dornfortsätze des I. bis I I. Rückenwirbels sind nach hinten gerichtet, der des letzten nach vorn. Der Dornfortsatz des I 2. Wirbels steht senkrecht, und wird dieser Rückenwirbel als der diaphragmatische bezeichnet. Mitunter steht bereits der Dornfortsatz des I I. Rückenwirbels senkrecht, was auch an einigen Schafskeleten der zoologischen Sammlung der Fall ist. Auch Franck $\left({ }^{3}\right)$ bezeichnet den I I. als den diaphragmatischen Wirbel für das Schaf, während H. von Nathusius ( $\left.{ }^{4}\right)$ sagt: ,dass aber auch schon der I I. Rückenwirbel entschieden der diaphragmatische sein kann, ist meines Wissens bisher noch nicht beobachtet worden." Giebel $\left({ }^{9}\right)$ bezeichnet den I 2. bis I3. Rückenwirbel als den diaphragmatischen für das Schaf. Müller ( $\left.{ }^{2}\right)$ giebt an, dass der I I. oder I2. der diaphragmatische sei; nach Martin's ( $\left.{ }^{8}\right)$ Ansicht ist es beim Schafe nur der I I. Rückenwirbel.

An den Dornfortsätzen hebt $H$. von Nathusius ( $\left.{ }^{4}\right)$ bei sämmtlichen Schafrassen als ein wesentliches. Merkmal hervor, dass an dem hinteren Rande der ersten 8 Rückenwirbel, unmittelbar über dem canalis columnae spinalis beginnend, eine mehr oder weniger deutliche Rinne vorhanden ist. Ich fand diese Rinne beim Marschschafe und bei der Heidschnucke am Dornfortsatze des 3. und 4. Rückenwirbels sehr deutlich ausgeprägt, an dem des 8. dagegen nur schwach angedeutet; vom I.-5. Rückenwirbel ist diese Rinne jedoch stets so tief, dass sie eine dreieckige, scharfrandige Grube bildet, welche den vorderen scharfen Rand jedes Dornfortsatzes des nachfolgenden Wirbels, besonders im unteren Drittel des Fortsatzes, vollständig einschliesst. Dieses Ineinandergreifen trägt nicht unwesentlich mit zu der festen Verbindung und Stärke der Wirbelsäule beim Schafe bei. Vom 9. Rückenwirbel ab wird der hintere Rand der folgenden Dornfortsätze beständig schärfer; die Processus spinales der beiden letzten Rückenwirbel sind zudem am breitesten und bilden so den Uebergang zu den Lendenwirbeln.

Betrachten wir bei der Ziege zunächst die ventrale Fläche der einzelnen Rückenwirbel, so finden wir, dass die Wirbelkörper seitlich zusammengedrückt sind, wodurch die Wirbel an sich schmäler erscheinen als beim Schafe. Dieser Umstand dürfte für die Formation des ganzen Brustkorbes, welcher bei der Ziege viel weniger tonnenförmig gestaltet ist als bei dem Schafe, mit bedingend sein. Die Gelenkfortsätze sind an sämmtlichen Rückenwirbeln der Ziege nicht so stark entwickelt als beim Schafe, zumal vom 6. Wirbel an sind dieselben nahezu um ein Viertel kürzer. Die Angabe Martin's $\left(^{8}\right)$, dass der vordere Rand der Dornfortsätze beim Schafe meist mehr geradlinig ist, mit Ausnahme der ersten Rückenwirbel, habe ich an den vorliegenden Skeleten bestätigt gefunden. Die Dornfortsätze der Ziege sind ferner schlanker und bestehen fast nur aus kompakter Knochensubstanz, wodurch sie fester erscheinen; ihr vorderer und hinterer Rand ist schärfer als bei dem Schafe. Bei letzterem Thiere sind die oberen Enden der Dornfortsätze vielfach brüchig und bröckelig, bei der Ziege dagegen stets fest und stark; niemals erscheint hier die Substantia spongiosa an den Spitzen wie dies beim Schafe meistens der Fall ist. Ausserdem sind alle Dornfortsätze bis zum I I. Rückenwirbel bei der Ziege weit mehr nach rückwärts 
gerichtet, was an den ersten acht am meisten in die Augen fällt. Dabei ist an sämmtlichen Dornfortsätzen das obere Ende, zumal bei dem Ziegenskelet Nr. I 568 sehr deutlich, nach vorn aufgebogen, obgleich es die Gesammtrichtung der Dornfortsätze nach rückwärts, d. h. bis zum diaphragmatischen Wirbel, mitmacht. An den beiden Ziegenskeleten ist der I 2. Rückenwirbel der diaphragmatische, was auch von Müller ( $\left.{ }^{2}\right)$, Franck ( $\left.{ }^{3}\right)$ und Martin ( $\left.{ }^{8}\right)$ übereinstimmend angegeben wird. Die rinnenartige Vertiefung am hinteren Rande der ersten 8 Dornfortsätze des Schafes ist bei der Ziege Nr. I568 nur ganz schwach entwickelt, bei der Ziege Nr. I 556 noch nicht einmal andeutungsweise vorhanden.

\section{Lendenwirbel.}

An den beiden Schafskeleten ist die Zahl der Lendenwirbel verschieden: Das Marschschaf hat deren 7, die Heidschnucke dagegen nur 6; jedoch ist die letztere Zahl nach Müller ( $\left.{ }^{2}\right)$ als Regel zu betrachten. Nathusius $\left({ }^{4}\right)$ giebt an, dass wahrscheinlich meistens 6 Lendenwirbel vorhanden sind; doch sind nach seiner Ansicht die verschiedenen Zahlen nicht Eigenthümlichkeiten der verschiedenen Rassen, sondern nur individuelle Verschiedenheiten. Betrachten wir die gesammte Lendenwirbelparthie, so fällt uns die gleiche Länge der 5 ersten Wirbel sofort in die Augen, während der letzte Lendenwirbel regelmässig um den vierten Theil kürzer ist als jeder vorhergehende. Anders verhält es sich mit der Breite; dieselbe nimmt vom ersten bis zum letzten Lendenwirbel beständig $\mathrm{zu}$, so dass der 6. bezw. der 7 . Wirbel der breiteste von allen ist. Meine Messungen ergeben folgende Zahlen:

$\begin{array}{lcccc} & \text { I. } & 6 . & 7 . & \\ \text { Marschschaf: } & 27 & 34 & 39 & \mathrm{~mm} \\ \text { Heidschnucke: } & \text { I } 9 & 26 & \text { fehlt } & \mathrm{mm}\end{array}$

Die Maasse sind an der ventralen Fläche der einzelnen Wirbelkörper unmittelbar am hinteren Rande der Basis der Querfortsätze, also an der schmalsten Stelle der Wirbel, aufgenommen. Die Breite der Dornfortsätze steht bei den Lendenwirbeln nicht in irgend einem Verhältniss zur Wirbellänge, sondern ist verschieden. Bei der kurzschwänzigen, ostfriesischen Tieflandsrasse varïrt die Breite sehr beträchtlich, da der Dornfortsatz des 3., 4. und 5. Lendenwirbels etwa um ein Drittel breiter ist als der Dornfortsatz der 3 übrigen Wirbel; bei der Heidschnucke ist dieser Unterschied nicht so bedeutend, jedoch ist auch hier der Dornfortsatz des letzten Lendenwirbels am schmalsten.

Nach H. von Nathusius ( $\left.{ }^{4}\right)$ verhält sich die Höhe der Dornfortsätze $\mathrm{zu}$ der des Körpers der Lendenwirbel bald wie I,5 : I, bald wie I,8 : I. Sieht man von hinten frontalwärts über die Dornfortsätze der ganzen Lendenwirbelparthie hinweg, so findet man, dass die oberen Ränder nahezu in einer Ebene liegen, was auch $\mathrm{H}$. von Nathusius ( ${ }^{4}$ ) hervorhebt. Ihrer Stellung nach erscheinen die Dornfortsätze des 2., 3. und 4. Lendenwirbels senkrecht zur Längsachse des Rückens, die übrigen sind mehr oder weniger nach vorn geneigt. 
Die Querfortsätze der Lendenwirbel (Tafel II., Fig. 7 und 8) weisen bei den einzelnen Rassen hinsichtlich der Form, Länge und Richtung einige Verschiedenheiten auf. Beim Marschschafe sind dieselben nahezu gleich breit, mit Ausnahme des letzten Lendenwirbels, der um ein Viertel schmälere Rippenfortsätze besitzt. Die des ersten und letzten Wirbels haben zudem eine von denen der übrigen abweichende Gestalt, und zwar in Folge der zitzenförmigen Ansätze, die sich beim ersten Lendenwirbel am vorderen, beim letzten dagegen am hinteren Rande der Querfortsätze befinden. (Tafel II., Fig. 7). Bei der Heidschnucke fehlen diese Ansätze und sind nur an den Querfortsätzen des ersten Lendenwirbels angedeutet.

Bei beiden Schafrassen laufen die dreikantig geformten Quer- oder Rippenfortsätze des letzten Lendenwirbels in eine Spitze aus und sind hakenartig stark nach vorn gekrümmt. Eine Theilung des letzten Querfortsatzes in zwei Enden ist nicht vorhanden, was auch Müller $\left(^{2}\right)$ als Abweichung von dem letzten Lendenwirbel des Rindes hervorhebt; bei dem Marschschafe könnte der zitzenförmige Ansatz an dem hinteren Rande des letzten Querfortsatzes vielleicht als das Rudiment einer zweiten Spitze aufgefasst werden. (Tafel II., Fig. 7.)

Auch die Länge der Querfortsätze ist bei den einzelnen Schafrassen etwas verschieden; die Gesammtlänge der einzelnen Lendenwirbel mitsammt ihren zugehörigen Rippenfortsätzen an den vorliegenden Skeleten gemessen, ergiebt nachstehende Zahlen in $\mathrm{mm}$ :

I. 2. 3. $4 . \quad$ 5. 6 .

Marschschaf: I o6, I 28, I 37, I 44, I 45, I 43

Heidschnucke: $69,8 \mathrm{I}, 87,85,85,78$

Der beim Marschschafe vorhandene 7. Lendenwirbel misst sogar nur I I $\mathrm{mm}$.

Nach H. von Nathusius ( $\left.{ }^{4}\right)$ ist die Richtung der Querfortsätze bei verschiedenen Thieren nicht ganz gleich, jedoch sind alle nach vorn gerichtet. Beim Marschschafe bilden die Querfortsätze des 4. bis 6 . Wirbels einen grösseren Winkel mit der Achse der Wirbelsäule als die der übrigen Lendenwirbel. Ausserdem bestehen noch Richtungsverschiedenheiten in Bezug auf ihre horizontale Lage; bei dem kurzschwänzigen, ostfriesischen Schafe finde ich, dass alle Querfortsätze ziemlich in einer Ebene liegen; bei der Heidschnucke sind die des ersten und letzten Wirbels etwas nach abwärts gerichtet, während alle der übrigen Lendenwirbel ebenso wie beim Marschschafe an den Enden mehr oder weniger stark nach aufwärts gebogen erscheinen.

Gleich wie die Rückenwirbel, so sind auch die Lendenwirbel bei der Ziege im Verhältniss zur Länge des Wirbelkörpers schmal und schlank, wodurch sie länger zu sein scheinen.

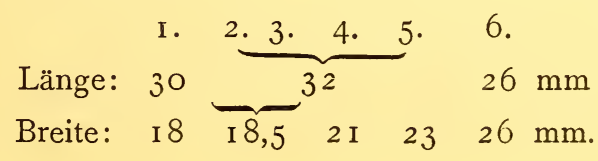


Mit Ausnahme des letzten Wirbels haben alle an der ventralen Fläche eine viel deutlicher ausgeprägte Knochenleiste als beim Schafe. Die Querfortsätze besitzen bei der Ziege eine etwas schräg von vorn und oben nach hinten und unten gestellte Basis; dieselben sind ferner ziemlich stark ventralwärts gerichtet, was auch $\mathrm{Martin}\left({ }^{8}\right)$ angiebt, niemals jedoch sind sie wie beim Schafe an den Enden wieder nach aufwärts umgebogen, sondern sie halten ihre ursprüngliche Richtung stets bei. Zudem sind die vorderen Ränder sämmtlicher Rippenfortsätze bei der Ziege mehr ausgehöhlt, und gleichzeitig sind die Enden der Querfortsätze schaufelartig verbreitert. Die kräftig entwickelten Dornfortsätze gehen bei der Ziege sämmtlich in eine mehr oder weniger starke, wulstig verdickte Leiste aus, welche frontalwärts über den Wirbelkörper bedeutend hinausragt. Die Angaben Martin's $\left.{ }^{8}\right)$, dass die Dornfortsätze der Lendenwirbel bei der Ziege mit Ausnahme der beiden ersten nahezu senkrecht stehen, habe ich an den in der zoologischen Sammlung vorhandenen Ziegenskeleten nicht bestätigt gefunden: Gleichwie beim Schafe so sind auch bei der Ziege die Dornfortsätze aller Lendenwirbel - die der ersten in geringerm, die der letzten in höherm Grade - schief nach vorn gerichtet. (Tafel II., Fig. 9.)

\section{Kreuzbein.}

Nach Müller ( ${ }^{2}$ ) und H. von Nathusius ( $\left.{ }^{4}\right)$ kann das Kreuzbein beim Schafe aus 3 bis 5 Wirbeln bestehen, in der Regel besteht es aus 4 Wirbeln. An den beiden zum Vergleiche gewählten Schafskeleten ist das Kreuzbein aus je drei mit einander verschmolzenen Wirbeln zusammengesetzt; dementsprechend sind auch 2 Paar obere sowie 2 Paar untere Kreuzbeinlöcher vorhanden. Länge und Breite dieser Wirbel stehen gewöhnlich in einem konstantem Verhältniss wie bei den Rückenwirbeln; für die Breite ist nur die der einzelnen Wirbelkörper, nicht aber die der Flügelfortsätze des ersten Kreuzwirbels massgebend. Die von mir ausgeführten Messungen ergaben folgende Zahlen:

Marschschaf:

Länge des Kreuzbeins: $77 \mathrm{~mm}$

I. 2. 3. Wirbel

Breite: I7 IO I $2 \mathrm{~mm}$;

Heidschnucke:

Länge des Kreuzbeins: $55 \mathrm{~mm}$

I. 2. 3. Wirbel

Breite: $\quad$ I3 15 I6 $\mathrm{mm}$.

Die ventrale Fläche des Kreuzbeins ist rundlich und in der Regel ganz glatt, jedoch von vorn nach hinten nicht stark konkav, weil nach Müller ( ${ }^{2}$ ) das Kreuzbein weniger stark gebogen ist als das des Rindes. Ein Promontorium am ersten Kreuzwirbel finde ich nur bei dem ostfriesischen Marschschafe angedeutet, bei der Heidschnucke ist die entsprechende Stelle durchaus eben und glatt.

Beide Schafrassen zeigen auch in der Bildung der Dornfortsätze des Kreuzbeins manche Verschiedenheiten: Bei dem Marschschafe finden 
wir alle vollständig mit einander verwachsen, was auch $\mathrm{H}$. von $\mathrm{Nathu-}$ sius $\left({ }^{4}\right)$ an vielen Skeleten gesehen hat; die Dornfortsätze bilden einen durchgehenden, hohen Kamm. Bei der Heidschnucke sind die Dornfortsätze von einander getrennt, sie stehen frei und sind - der erste nur schwach, die beiden letzten stark - nach hinten gerichtet.

Die Flügelfortsätze des Kreuzbeins der beiden Schafrassen gleichen sich in jeder Beziehung.

Franck $\left(^{3}\right)$ giebt an, dass auch der 2. Kreuzwirbel, wie bei dem Zackelschaf der Münchener Sammlung, mit an der Bildung der Flügelfortsätze betheiligt sein kann.

Die Zahl der Wirbel, aus welchen das Kreuzbein der Ziege zusammengesetzt ist, variirt wie beim Schafe, jedoch mit dem Unterschiede, dass niemals drei Kreuzwirbel allein vorkommen, sondern meistens 4, mitunter 5, was auch Franck $\left({ }^{3}\right)$ angiebt. Das Ziegenskelet Nr. I 556 zeigt 4, Nr. 1568 dagegen 5 Wirbel und dementsprechend auch 3 bezw. 4 Paar Foramina sacralia. Bei der Ziege beträgt die

Länge des Kreuzbeins: $82 \mathrm{~mm}$,

Breite des I. 2. 3. 4. Wirbels:

I3 $98 \quad 8 \quad 8 \quad \mathrm{~mm}$.

Bemerkenswerth erscheint es, dass bei der Ziege niemals an der ventralen Fläche des ersten Kreuzwirbels ein Promontorium angedeutet ist, wie bei einzelnen Schafrassen; die betreffende Stelle ist stets glatt und eben. Bei der Ziege sind ferner die lateralen Ränder der verwachsenen Wirbelkörper dünn und scharf; beim Schafe dagegen immer wulstförmig verdickt.

Die Dornfortsätze der ersten drei Kreuzwirbel sind bei allen in der Sammlung vorhandenen Ziegenskeleten vollständig mit einander verwachsen; der Dornfortsatz des letzten Wirbels allein steht frei und fällt stark nach hinten $a b$, was in geringem Grade bereits an dem Dornfortsatze des vorletzten Kreuzwirbels zu bemerken ist. In Folge dessen erscheint das Kreuzbein der Ziege an sich schon abschüssiger als das des Schafes, wozu noch bei ersterem Thiere die etwas stärkere Konkavität der ventralen Kreuzbeinfläche hinzukommt. (Tafel II., Fig. I2.)

\section{E. Schwanzwirbel.}

Beim Schafe ist nach H. von Nathusius $\left({ }^{4}\right)$ die Anzahl der Schwanzwirbel innerhalb der Art sehr verschieden, bei den einzelnen Rassen dagegen durchaus konstant, weshalb auch von jeher in der systematischen Eintheilung der Schafrassen zwischen kurz- und langschwänzigen Schafen unterschieden wird. Entgegengesetzte Behauptungen, wie solche von Settegast $\left({ }^{7}\right)$ aufgestellt wurden, sind nach $H$. von $\mathrm{Nathusius} \mathrm{noch} \mathrm{nicht} \mathrm{bewiesen} \mathrm{worden.}$

Von den beiden vorliegenden Skeleten besitzt das des ostfriesischen Marschschafes I 5, das der Heidschnucke i4 Schwanzwirbel, jedoch ist bei beiden der letzte Wirbel in gleicher Weise bis auf die Hälfte verkümmert und endigt mit einer Spitze; dabei sind die drei letzten 
Schwanzwirbel jedesmal mit einander verwachsen. Haemalbögen fehlen, was auch nach H. von Nathusius ( ${ }^{4}$ ) beim Schafe stets der Fall ist. Die vier ersten Schwanzwirbel zeigen noch einen geschlossenen Wirbelkanal, an den übrigen ist ein solcher nicht mehr vorhanden. Die Länge der einzelnen Wirbel nimmt bis zum Io. allmählich, alsdann jedoch stärker ab, sodass der I4. beim Marschschafe nur halb so lang ist als der erste; ganz analog verhält sich die Abnahme der Breite der einzelnen Schwanzwirbel.

Die Dornfortsätze sind bis zum 4. Wirbel nachzuweisen, die Querfortsätze noch bis zum 7.; H. von Nathusius $\left(^{4}\right)$ giebt an, dass bei den langschwänzigen Schafrassen die Querfortsätze dagegen noch bis zum I I., sogar mitunter bis zum I 2. Schwanzwirbel, natürlich allmählich schwächer werdend, erkennbar sind.

Bei der Ziege beträgt die Anzahl der Schwanzwirbel in der Regel I 2, jedoch sind die einzelnen Wirbelkörper verhältnissmässig etwas kürzer als beim Schafe. Die ebenfalls bis zum 7. Schwanzwirbel nachweisbaren Querfortsätze sind bei der Ziege flacher und mehr schaufelförmig verbreitert.

\section{F. Rippen.}

Ebenso wie die Rippen bei den einzelnen Schafrassen, abgesehen von den Grössendimensionen, nur geringe Verschiedenheiten darbieten, so sind letztere auch zwischen Schaf und Ziege nicht in hohem Grade hervorstechend.

Bei beiden Thierarten sind entsprechend der Anzahl der Rückenwirbel I3 Rippen vorhanden, nämlich 8 wahre und 5 falsche. Länge und Breite, sowie Dicke und Krümmung der Rippen bieten einige wichtige Erkennungsmerkmale zwischen Schaf und Ziege dar. Die Rippen der Ziege sind zunächst verhältnissmässig länger und auf dem Querschnitt rundlicher als beim Schafe; dadurch erscheinen sie etwas stärker als die mehr flachen Rippen des Schafes. Die bedeutendere Breite des unteren Endstückes bei der Ziege zeigt sich am besten an der ersten Rippe. Die Krümmung der Rippen ist bei der Ziege bei weitem stärker; Martin ( $\left.{ }^{8}\right)$ sagt deshalb sehr treffend: „Die Rippen der Ziege sind mehr S-förmig geschwungen."

\section{G. Brustbein.}

Die am Brustbein des Schafes und der Ziege vorhandenen Unterschiede sind nach Martin $\left(^{8}\right)$ im Allgemeinen so gering, dass eine Auseinanderhaltung schwer möglich ist. Als einzige Unterscheidungsmerkmale, welche ich an den vorliegenden Skeleten auffand, möchte ich jedoch nicht unerwähnt lassen, dass die einzelnen das Brustbein bildenden Stücke bei der Ziege verhältnissmässig schmäler sind, zudem dass das Brustbein an der unteren Fläche ziemlich stark konkav ist; beim Schafe dagegen erscheint diese Fläche breit und flach. H. von

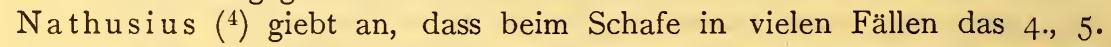
und 6. Brustbeinstück, auch noch im höchsten Alter, median und meistens 
asymmetrisch getheilt ist: An dem Skelete der Heidschnucke ist dies beim 6. Brustbeinstück der Fall, dagegen an keinem der im zoologischen Museum vorhandenen Ziegenskelete.

\section{H. Becken.}

Nach H. von Nathusius ( $\left.{ }^{4}\right)$ kommt beim Becken des Schafes die geschlechtliche Differenz, gleichwie auch bei den anderen Säugethieren, wesentlich in Betracht, da das Becken des männlichen Thieres von dem des weiblichen konstant verschieden ist. Für die Zwecke der vergleichenden Osteologie des Schafes und der Ziege ist es daher nothwendig, ausschliesslich Beckenknochen entweder von männlichen oder von weiblichen Thieren zur Untersuchung zu wählen: Die vorliegenden Skelete stammen sämmtlich von weiblichen Thieren.

Meine Messungen an den einzelnen Becken der beiden Schafe sowie der Ziege ergeben folgende Zahlen; dabei muss bemerkt werden, dass zur Feststellung der Länge jede Beckenhälfte von dem vorderen Rande des äusseren Darmbeinwinkels bis zum äussersten Punkte des Sitzbeinhöckers, sowie zur Feststellung der Breite am ganzen Becken der Abstand zwischen den lateralen Pfannengelenksrändern gemessen wurde:

a) Marschschaf:

Länge: $24,8 \mathrm{~cm}$

Breite : I $3,5 \mathrm{~cm}$

Länge der Schambeinfuge $5,6 \mathrm{~cm}$;

b) $\mathrm{Heidschnucke:}$

Länge: $16,2 \mathrm{~cm}$

Breite: $9,3 \mathrm{~cm}$

Länge der Schambeinfuge: $4,5 \mathrm{~cm}$;

c) Ziege Nr. I568:

Länge: $23,2 \mathrm{~cm}$

Breite: IO, I cm

Länge der Schambeinfuge: $6,8 \mathrm{~cm}$.

Aus diesen Zahlen ergiebt sich, dass das Becken der Ziege bedeutend länger und schmäler, und dass die Beckenöffnung beträchtlich enger ist als beim Schafe; hierauf beruht auch die viel geringere Breite der ganzen Kruppenparthie bei der Ziege. Der Beckenausgang erscheint beim Schafe mehr rund, bei der Ziege mehr länglich rund.

Die einzelnen das Becken bildenden Knochen, das Sitzbein, das Schambein, besonders aber das Darmbein, sind bei der Ziege schlanker; dies macht sich nicht allein an der Säule, sondern auch an der Schaufel des Darmbeins bemerkbar. (Tafel III., Fig. I3-15.) Am auffallendsten ist jedoch die erhebliche Länge der Symphyse des Schambeins bei der Ziege im Gegensatze zum Schafe. Die Sitzbeinhöcker liegen bei letzterem Thiere, entsprechend der weiteren Beckenöffnung mehr auseinander und sind zudem auch flacher gestellt; in Folge dessen bildet der hintere Sitzbeinausschnitt (arcus ossium pubis h.) bei der Ziege einen bei weitem spitzeren Winkel. 
H. von Nathusius sagt mit Recht, dass uns in Bezug auf den Sitzbeinhöcker die vom Pferde abgeleitete Nomenklatur beim Schafe einigermassen im Stiche lässt; es findet sich nämlich nach seiner Angabe beim Schafe an dem äusscren, vorderen Rande des Sitzbeinhöckers ein rechtwinkelig auf die Längenachse des Rumpfes und mit der Spitze nach unten gerichteter Fortsatz. Letzterer ist an den beiden vorliegenden Schafskeleten, zumal an dem Sitzbeinhöcker des Marschschafes, sehr stark entwickelt, nach unten gerichtet und quergestellt; bei der Ziege beträgt seine Länge verhältnissmässig kaum die Hälfte von der Länge desselben Fortsatzes beim Schafe; ausserdem ist hier dieser Fortsatz des Sitzbeinhöckers nach vorn und oben gebogen. Schliesslich möchte ich noch erwähnen, dass das Foramen obturatorium bei dem Schafe bedeutend kürzer und rundlicher gestaltet ist als bei der Ziege, was auch Martin ( $\left.{ }^{8}\right)$ hervorhebt. (Tafel III., Fig. I3-I 5.)

\section{J. Schädel.}

Für das vorliegende Thema dürfte es am zweckmässigsten sein, den Schädel des Schafes und der Ziege als Ganzes betrachtet miteinander zu vergleichen.

Bei einer Vergleichung fällt zunächst die Mannigfaltigkeit auf, welche durch die Abwesenheit oder das Vorhandensein von Hörnern bedingt ist. Es giebt bekanntlich sowohl unter den Schafen als auch unter den Ziegen gehörnte und ungehörnte Rassen; je nachdem Hornfortsätze fehlen, oder solche mehr oder weniger stark entwickelt sind, hat der Schädel des Schafes und der Ziege eine sehr verschiedene Form. Gleichzeitig kommt hierbei auch noch die Form und Richtung der Hörner bei den gehörnten Thieren in Betracht. Diese durch die Entwicklung der Hörner bedingte verschiedene Schädelformation betrifft vorwiegend das Stirnbein. Nach Müller $\left({ }^{2}\right)$ sind die Hornfortsätze beim Schafe durch einen längeren, bei der Ziege durch einen kürzeren Zwischenraum, zu dessen Herstellung die beiderseitigen Stirnbeine in gleicher Weise beitragen, von einander getrennt. Dieser Zwischenraum erscheint beim Schafe stets rundlich und breit, bei der Ziege dagegen spitzwinklig und schmal, weil bei ersterem Thiere die Hornfortsätze mehr oder weniger weit nach aussen gerückt, bei letzterem dagegen der Mittellinie des Schädels genähert sind. Die Hornfortsätze selbst sind bei der Ziege, was auch Franck (3) angiebt, seitlich zusammengedrückt und auf den beiden, einander zugekehrten, medianwärts gerichteten Flächen fast vollständig flach, niemals aber rundlich auf dem Querschnitte wie beim Schafe. Auch Zittel $\left({ }^{10}\right)$ giebt an, dass bei der Ziege die Hornzapfen seitlich komprimirt, im Durchschnitt länger als breit und vorne mit einer Kante versehen, die des Schafes aber mehr dreikantig, vorne breit und im Dnrchschnitt breiter als lang sind.

Sehr charakteristisch ist meist auch die Verschiedenheit der Profillinie bei beiden Thierarten, welche durch die Richtung der Stirn- und Nasenbeine bedingt ist. Bei dem kurzschwänzigen, ostfriesischen Schafe stellt die Profillinie einen ziemlich stark konvexen, bei der Ziege, besonders bei dem Ziegenskelet Nr. I 568, dagegen einen konkaven Bogen 
dar. Jedoch kommen hierbei, wie überhaupt am ganzen Schädel sowohl beim Schafe als auch bei :der Ziege manche Rasseneigenthümlichkeiten vor, wodurch die Formation des Kopfes vielfach variirt erscheint.

Nach Müller $\left(^{2}\right)$ ist die dorsale Fläche des Schädels, zu deren Bildung auch die Scheitelbeine mit beitragen, beim Schafe je nach den Rassen mehr oder weniger stark gewölbt; bei der Ziege ist dies nur in ganz geringem Grade der Fall, was sich auch an allen in der zoologischen Sammlung vorhandenen Skeleten sehr deutlich ausspricht. Nach Zittel $\left({ }^{10}\right)$ ist der Schädel des Schafes breit, die Nasenbeine kurz und breit, dagegen der Schädel der Ziege schmal, sowie auch die Nasenbeine lang und schmal.

Beim Schafe kommt an der Gesichtsfläche eine mitunter seichte, bei einigen Rassen z. B. bei der ostfriesischen Tieflandsrasse ziemlich tiefe Grube vor, welche unterhalb und, nach der Medianebene des Kopfes zu, vor der Augenhöhle ihre Lage hat; dieselbe heisst die äussere Thränengrube und wird vom Thränen- und Jochbein gebildet. Diese Grube fehlt bei der Ziege vollständig. Schliesslich sei noch erwähnt, dass nach Franck $\left(^{3}\right)$ der Gesichtswinkel beim entwickelten Schafe $127^{\circ}$, bei der Ziege annähernd $125^{\circ}$ beträgt.

\section{K. Knochen der vorderen Gliedmaassen.}

Das Schulterblatt der Ziege ist verhältnissmässig etwas länger, gleichzeitig aber auch bedeutend schmäler als das des Schafes, was nachstehende Messungsergebnisse zeigen:

a) Marschschaf:

Länge des Schulterblattes: $18 \mathrm{~cm}$

Breite " " I $4 \mathrm{~cm}$;

b) Heidschnucke:

Länge des Schulterblatts: I $2,6 \mathrm{~cm}$

Breite " , : $7,8 \mathrm{~cm}$;

c) Zi ege:

Länge des Schulterblatts: $17,8 \mathrm{~cm}$

Breite ", " : $9,7 \mathrm{~cm}$.

Auch Murie (11) weist durch Zahlen nach, dass das Schulterblatt des Schafes relativ breiter ist als das der Ziege; er hat seine Untersuchungen an dem Skelet einer Saiga-Antilope gemacht.

Besondere Unterschiede zeigt ferner noch die Gräte des Schulterblatts: Beim Schafe beginnt dieselbe unmittelbar am oberen Rande, dicht unter der Insertion des Schulterblattknorpels; sie steigt rasch steil an und bildet in der Mitte eine wulstartige Verdickung, welche im Bogen etwas nach hinten geworfen erscheint, was auch Martin ( $\left.{ }^{(}\right)$ beobachtet hat. Bei der Ziege dagegen nimmt die Gräte mehr unterhalb des Ansatzes des Schulterblattknorpels ihren Anfang, sie hat einen ziemlich flachen, durchaus geraden Verlauf und erreicht verhältnissmässig nur zwei Drittel der Höhe der Gräte beim Schafe. Bei der 
Ziege ist zudem noch das Collum scapulae, der schlankeren Form des ganzen Knochens gemäss, deutlicher ausgeprägt, was an beiden Ziegenskeleten, namentlich aber an dem Schulterblatte eines vier Tage alten Ziegenböckchens, dessen Skelet von mir präparirt wurde, besonders schön zu sehen ist. (Tafel III., Fig. I6-I8.)

Von den übrigen Knochen bieten nur noch der Humerus und der Metacarpus an der Vorder-Extremität bei Schaf und Ziege Verschiedenheiten dar. Das Armbein ist bei der Ziege länger, der Metacarpus aber kürzer als beim Schafe, was ich bei sämmtlichen im zoologischen Museum vorhandenen Skeleten (von diesen beiden Thierarten) beobachten konnte: Diese Unterschiede sind so bedeutend, dass sie allein schon eine genaue Unterscheidung zwischen den diesbezüglichen Knochen der Ziege und des Schafes geben dürften, da es sich dabei also nicht um Rassenunterschiede handeln kann.

Hinsichtlich der Länge der erwähnten Knochen habe ich folgende Maasse aufgezeichnet:

a) Marschschaf:

Humerus: I 5,6 Metacarpus: I 5,2 cm,

b) Heidschnucke:

Humerus: I I,4 Metacarpus: I I,9 cm,

c) Ziege:

Humerus: i6,6 Metacarpus I 2,8 cm.

Das Armbein ist beim Schafe zudem auch gedrungener und weist, wie auch Martin $\left(^{8}\right)$ angiebt, eine stärkere spiralige Windung auf als bei der Ziege. Die schlankere Form des Armbeins bei letzterem Thiere kommt ausser an dem Mittelstück auch an den beiden Epiphysen dieses Knochens zum Ausdruck; sowohl die drei Furtsätze am oberen Endstück, der Gelenkkopf, der starke, laterale wie auch beim Rinde den Gelenkkopf bedeutend überragende Fortsatz, sowie der mediale Fortsatz, als auch am unteren Endstück die Gelenkrolle mit den hinter derselben gelegenen Armbeinknorren sind bei der Ziege zierlicher und feiner entwickelt.

Bei ungefähr gleicher Länge des Radius und der Ulna bei beiden Thierarten sind diese Knochen bei der Ziege gleichfalls schlanker und dünner als beim Schafe. Müller ( $\left.{ }^{2}\right)$ giebt an, dass bei dem Schafe und der Ziege das sehr dünn ausgebildete Griffelbein mitunter vollständig fehlt oder durch einen Kamm am äusseren Rande ersetzt wird. An den vorliegenden Skeleten, welche sehr sorgfältig präparirt sind, ist ein solches Griffelbein beim Schafe stets vorhanden. Nach Franck's $\left({ }^{3}\right)$ Angabe fehlt das Griffelbein bei der Ziege gänzlich; H. von Nathusius berichtet dagegen, dass er bei der hiesigen Ziege sogar bis $25 \mathrm{~mm}$ lange Griffelbeine gefunden habe. Auch das Ziegenskelet Nr. I 568 besitzt ein $23 \mathrm{~mm}$ langes Griffelbein, eine Thatsache, die $\mathrm{H}$. von Nathusius im Inventarverzeichniss der Hundisburger Sammlung durch den Zusatz folgender Worte besonders betont: „Zum ersten Mal an einer Ziege gesehen! fehlt also nicht!“ 


\section{Knochen der hinteren Gliedmaassen.}

Von den Knochen der Hinterextremität bieten Femur und Tibia bei den einzelnen Schafrassen nach $H$. von Nathusius ( $\left.{ }^{4}\right)$ keine wesentlichen Unterschiede dar. Vergleichen wir aber das Ober- und Unterschenkelbein von Schaf und Ziege mit einander, so finden wir zunächst ein verschiedenes Längenverhältniss zwischen diesen Knochen: Femur und Tibia sind bei der Ziege verhältnissmässig kürzer, was auch nachstehende Zahlen, welche die Länge dieser Knochen angeben, beweisen:

a) Marschschaf:

Femur: 2 I,2 Tibia: $23,5 \mathrm{~cm}$,

b) H eidschnucke:

Femur: 1 5,4 Tibia: I 7,9 cm,

c) Z i ege Nr. I568:

Femur: I9,8 Tibia: 22,5 cm.

Am Oberschenkelbein habe ich die Achse zwischen dem äusseren Rande des Gelenkkopfes und der Tangente des medialen Gelenkhöckers, am Unterschenkelbein die Achse zwischen der Höhe der medialen Gelenkfläche am oberen und dem Malleolus internus am unteren Ende des Knochens gemessen.

H. von Nathusius ( ${ }^{4}$ ) ist auch der Ansicht, dass der Oberschenkel der Ziege relativ kürzer sei als der des Schafes und fügt hinzu, dass dies auch im Verhältniss zur Länge des Oberarms stehe. Martin $\left(^{8}\right)$ giebt an, dass die Tibia des Schafes im oberen Theile oft, nicht immer, etwas schmäler sei und sich nach unten verbreitere. An den vorliegenden Skeleten erscheint das Oberschenkelbein der Ziege schlanker und dünner, die Fortsätze feiner, auch zeigt sich der Hals des Gelenkkopfes bei weitem schärfer ausgeprägt als bei dem Schafe; durch letzteren Umstand tritt der Gelenkkopf bei der Ziege ziemlich stark hervor. Die Beobachtungen Martin's $\left(^{8}\right)$, dass die Kämme der Rolle am Femur des Schafes im Verhältniss zu ihrer Länge weiter auseinander stehen, habe ich an den in der zoologischen Sammlung vorhandenen Schafskeleten bestätigt gefunden.

Die Tibia hat bei der Ziege nahezu einen ganz geraden Verlauf, während sie beim Schafe - besonders im mittleren Theile der Diaphyse am stärksten — über die Fläche gebogen ist; die Konkavität liegt an der hinteren Fläche des Knochens. Ausserdem ist das Unterschenkelbein beim Schafe stärker spiralig gedreht als bei der Ziege.

Die Fibula fehlt nach $\mathrm{H}$. von Nathusius bei dem Schafe nicht so häufig, als gewöhnlich angenommen wird; auch das Skelet des kurzschwänzigen, ostfriesischen Tieflandsschafes (Tafel V., Fig. 26) weist eine deutlich entwickelte, nahezu bis zur Mitte der Tibia reichende und daselbst mit letzterem Knochen verwachsene Fibula auf. An den sämmtlichen in der zoologischen Sammlung vorhandenen Ziegenskeleten dagegen fehlt das Wadenbein. (Tafel V., Fig. 30.) 
An den Tarsalknochen habe ich ebensowenig wie an den Carpalknochen bei Schaf und Ziege für vorliegenden Zweck verwerthbare Unterscheidungsmerkmale auffinden können; abgesehen vom Metatarsus muss ich dies auch von den übrigen Knochen der Hinterextremität sagen.

Der Metatarsus ist, wie auch nachstehende Maasse zeigen, wieder bei der Ziege relativ etwas kürzer als beim Schafe.

Länge des Metarsus:
a) Marschschaf: $\mathrm{I}_{5, \mathrm{I}} \mathrm{cm}$
b) Heidschnucke: i i,6,
c) Ziege:
I 3 "

Ferner ist dieser Röhrenknochen bei der Ziege ziemlich schlank und auf dem durch die Diaphyse gelegten Querschnitte rundlich, beim Schafe dagegen verhältnissmässig breit und auf dem Querschnitte länglich. (Tafel V, Fig. 26, 28 und 30.)

\section{Knochen von Föten und jugendlichen Thieren.}

Aus der zoologischen Sammlung des Museums für Naturkunde zu Berlin wurden mir zwei Skelete von Schafföten (ovis aries), sowie ein Skelet von dem Fötus einer Ziege (capra mambrica) leihweise überlassen, welche ich bei meinen Untersuchungen mit zum Vergleiche heranzog. $\mathrm{Zu}$ demselben Zwecke habe ich im zoologischen Institut zu Berlin mehrere Skelete von Schaf- und Ziegenlämmchen im Alter von 4 bis 6 'Tagen präparirt und dabei Folgendes ermittelt.

An beiden Föten von ovis aries finde ich die Verbindungsstücke an den Bogen der Halswirbel noch nicht verknöchert, sondern noch knorpelig vorgebildet. Ferner besteht das Mittelstück am Arcus anterior des Atlas noch aus Knorpel; ebenso verhält es sich mit den Flügelfortsätzen des I. sowie dem Kamm des 2. Halswirbels. An dem Skelete des Ziegenfötus, welcher beinahe schon die vollständige Ausbildung erlangt hatte, sind die genannten Theile bis auf die Flügelfortsätze des Atlas und die Querfortsätze des 3. bis 6. Halswirbels bereits verknöchert; diese bestehen noch aus Knorpelsubstanz.

Die an den Föten gemachten Beobachtungen stimmen mit den Angaben Bonnets (12) übernin, welcher fand, dass die verknorpelten Wirbelbogen mit dem Wirbelkörper verschmelzen und so die Seitentheile des dorsal noch offenen Wirbels bilden. Bei den zur Untersuchung gezogenen Wirbeln ganz junger, aber bereits geborener Thiere, ist allerdings das Stadium der Verknöcherung schon weiter vorgeschritten, doch lässt sich bei den Schafföten erkennen, dass die Wirbel von je einem Knochenkern in der Basis der beiden Neuralbogenhälften und von einem in der Mitte des Wirbelkörpers aus verknöchern.

Gleichwie an den ausgebildeten Individuen sehen wir auch an den Föten als charakteristische Unterschiede zwischen Schaf und Ziege, dass die knorpelige Anlage der Flügelfortsätze des Atlas bei dem Ziegenfötus bedeutend länger ist, als bei denen des Schafes, ferner dass die 
Dornfortsätze des dritten bis letzten Halswirbels bei den Schafföten nur angedeutet, bei dem Fötus von der Ziege dagegen, ganz besonders am 5., 6. und 7. Halswirbel, deutlich entwickelt sind. Bei letzterem ist am Körper des 2. bis 4. Halswirbels auch eine Gräte nachzuweisen, welche bei den Schafföten fehlt.

Von den übrigen Theilen der Wirbelsäule muss zunächst erwähnt werden, dass die Dornfortsätze der vier letzten Rückenwirbel, sowie auch die aller Lenden- und Kreuzwirbel bei den Schafföten in ihrer knorpeligen Anlage verhältnissmässig niedriger und schwächer erscheinen als die des Ziegenfötus.

Hinsichtlich der Querfortsätze der Lendenwirbel bestehen in Form, Länge und Richtung bei den Föten und Jugendformen einer jeden der untersuchten Thierspecies die gleichen Unterschiede, welche ich bei den ausgewachsenen Individuen beobachten konnte. Andeutungen von Rippenrudimenten an den Lenden- und Kreuzwirbeln, wie solche von Bonnet ( ${ }^{12}$ ) bei den fötalen Formen von Pferd, Schwein und Schaf vermuthet werden, sind an den vorliegenden Föten nicht vorhanden.

Beim Ziegenfötus fällt die bedeutende Breite namentlich des unteren Drittels der wahren Rippen sofort in die Augen; desglcichen am Schulterblatt die erheblichere Länge desselben im Gegensatz zu dem der beiden Schafföten. Auch ist das Schulterblatt des Ziegenfötus im Ganzen schmäler, was sich am deutlichsten durch den weit geringeren Abstand des vorderen und hinteren Schulterblattwinkels zu erkennen giebt; zudem ist die Gräte bei dem Fötus der Ziege nicht so lang und hoch wie bei denen des Schafes. Von den vier Knochenstücken der Scapula, welche von ebensovielen Ossifikationspunkten aus während der Embryonalzeit gebildet werden, sind die Beule und der bei den Wiederkäuern rudimentär vorhandene Rabenschnabel-Fortsatz bei dem Ziegenfötus, sogar auch noch bei den bereits mehrere Tage alten Schaf- und Ziegenlämmchen, deutlich von einander gesondert; bei den viel jüngeren Schafföten sind diese Theile noch knorpelig. Der nach Bonnet (12) als unverknöcherter Rest der knorpeligen Schulterblattanlage aufzufassende Schulterblattknorpel ist bei dem Ziegenfötus verhältnissmässig grösser und umfangreicher als bei den Schafföten.

Die verschiedenen Längendimensionen des Humerus und Metacarpus, sowie die verhältnissmässig gleiche Länge des Radius beider Thierarten finden wir auch bei den Föten von Schaf und Ziege wieder; nur sind hier Radius und Ulna relativ etwas länger als bei den ausgebildeten Thieren.

Während beim Rinde noch eine vollständig entwickelte Ulna vorhanden und am unteren Endstück mit dem Radius durch Synostosis verwachsen ist, reducirt sich nach Bonnet ( ${ }^{12}$ ) die Ulna bei Schaf und Ziege dagegen etwas weiter und verwächst im Alter mit dem Radius in grösserer Ausdehnung. Ich möchte nicht unerwähnt lassen, dass von den vorliegenden Fötalskeleten das Ellbogenbein bei der Ziege, auch noch bei dem vier Tage alten Ziegenböckchen, fast vollständig von der Speiche getrennt, bei den viel jüngeren Schafföten dagegen bereits mit letzterem Knochen verwachsen ist. Die Ulna selbst ist bei den Föten 
beider Thierarten in verhältnissmässig gleicher Länge wie bei den erwachsenen Individuen entwickelt; nur das distale Ende derselben erreicht die Länge des Radius nicht, sondern bleibt etwa um den zehnten Theil hinter der Länge dieses Knochens zurück.

Auch an dem Becken der erst mehrere Tage alten Ziegen finde ich gleich wie bei den Föten das Sitzbein mit dem Schambein bereits verwachsen, von dem Darmbein jedoch durch eine Knorpelschicht getrennt. Die Knochen der Beckengliedmaassen zeigen bei den Jugendformen einer jeden Thierspecies ungefähr dieselben Verschiedenheiten bezw. die Anlage zu denselben wie bei den ausgebildeten Thieren; nur ist bei dem Fötus der Ziege die Tibia verhältnissmässig etwas länger als bei dem erwachsenen Individuum.

Während bei den ziemlich jungen Schafföten die Epiphysen an sämmtlichen Röhrenknochen noch knorpelig sind, zeigen sich beim Ziegenfötus an jedem Knochen die Epiphysen, wenn auch noch nicht wie die Diaphyse, verknöchert, aber noch durch starke Knorpelscheiben von einander getrennt, wodurch die drei typischen Ossificationsstellen der Röhrenknochen deutlich charakterisirt werden.

An den vorliegenden Föten und Jugendformen von Schaf und Ziege bieten alle übrigen Theile des Skelets gleiche Verhältnisse dar, wie die Knochen der ausgebildeten Thiere.

Zum Schlusse sei es mir gestattet, die wichtigsten osteologischen Unterschiede zwischen Schaf und Ziege noch einmal in Kürze zusammenzufassen.

Der Atlas der Ziege ist länger und schmäler als der des Schafes, das Tuberculum anterius ist höher und schärfer ausgeprägt und bildet, in den vorderen Ausschnitt zwischen den Gelenkfortsätzen auslaufend, einen starken, zahnförmigen Vorsprung, wodurch der vordere Ausschnitt in zwei scharfgetrennte, halbmondförmige Ausschnitte zerfällt. Zudem sind die Flügelfortsätze bei der Ziege bedeutend länger als beim Schafe.

Auch der Epistropheus ist bei weitem schmäler und schlanker bei der Ziege, sein Kamm ist länger und ragt sowohl nach vorn als auch nach hinten beträchtlich über den Wirbelkörper hinaus, mit dem er in Folge dessen viel schärfere Ausschnitte an beiden Enden bildet, als dies beim Schafe der Fall ist. Bei der Ziege fehlt das Querfortsatzloch am Epistropheus, findet sich dagegen am 3. bis 6. Halswirbel gleichwie beim Schafe; das Zwischenwirbelloch ist jedoch um die Hälfte grösser als bei letzterem Thiere.

Die 5 letzten Halswirbel haben bei der Ziege längere, spitzere und schartkantigere Dornfortsätze als beim Schafe; ganz besonders gilt dies vom 7. Halswirbel. Die Dornfortsätze der Ziege laufen in eine Spitze aus, endigen niemals breit, wie beim Schafe und sind auch mehr schief nach vorn umgelegt. Die Querfortsätze sind dünner und zierlicher; am 2., 3., 4. und 5. Halswirbel besitzt die ventrale Fläche des Körpers eine schärfere Knochenleiste als beim Schafe.

Auch die Rückenwirbel der Ziege sind verhältnissmässig schmäler, ihre Gelenkfortsätze sind nicht so stark entwickelt als beim Schafe; die 
Dornfortsätze sind schlanker und am oberen Ende nach vorn aufgebogen, der vordere und hintere Rand derselben ist sehr scharf. Die dreieckige, rinnenartige Vertiefung an dem hinteren Rande der ersten 5-8 Dornfortsätze des Schafes fehlt bei der Ziege. Während beim Schafe der I I. oder der I 2. Rückenwirbel der diaphragmatische sein kann, ist es bei der Ziege ausschliesslich der I2. Rückenwirbel.

Die Lendenwirbel besitzen bei der Ziege eine erheblichere Länge und schlankere Form als beim Schafe; dies prägt sich hauptsächlich an den einzelnen Wirbelkörpern aus, welche, mit Ausnahme des letzten Wirbels, an ihrer ventralen Fläche eine stärkere Knochenleiste erkennen lassen. Abweichend vom Schafe bilden die Dornfortsätze an den Lendenwirbeln der Ziege eine wulstartig verdickte Leiste am oberen Ende, welche nach vorn über den Wirbelkörper hinausragt. Die Basis der Querfortsätze ist bei der Ziege etwas schräg von vorn und oben nach hinten und unten gerichtet; dieselben verlaufen ventralwärts bis zu ihren Endpunkten hin, während sie beim Schafe an den Enden nach aufwärts umgebogen sind. An den vorderen Rändern erscheinen die Querfortsätze bei der Ziege stärker ausgehöhlt, sowie an den Enden schaufelartig verbreitert.

Die Zahl der Kreuzwirbel beträgt bei der Ziege wenigstens 4, niemals ist das Kreuzbein nur aus 3 Wirbeln zusammengesetzt, wie dies beim Schafe der Fall sein kann. Das Promontorium fehlt bei der Ziege; die lateralen Ränder der verwachsenen. Kreuzbeinwirbel sind hier dünn und scharf, beim Schafe wulstig verdickt. Das Kreuzbein der Ziege hat eine mehr abschüssige Form.

Bei der Ziege ist die Zahl der Schwanzwirbel stets geringer, auch sind die einzelnen Wirbelkörper relativ kürzer als beim Schafe.

Die das Brustbein zusammensetzenden Stücke sind bei der Ziege etwas schmäler; die untere Fläche derselben erscheint hier konkav, beim Schafe dagegen flach und eben.

Das Becken der Ziege ist erheblich schmäler und länger, und in Folge dessen die Beckenöffnung bedeutend enger als beim Schafe; Darmbein, Schambein und Sitzbein erscheinen schlanker und dünner, die Symphysis pelvis ist beträchtlich länger. Die Sitzbeinhöcker liegen beim Schafe weiter auseinander und sind flacher gestellt; der hintere Sitzbeinausschnitt bildet bei der Ziege einen kleineren Winkel. Der am vorderen Rande des Sitzbeinhöckers beim Schafe vorhandene Fortsatz ist bei der Ziege kaum halb so lang, sowie nach vorn und oben gebogen, beim Schafe dagegen quer gestellt und nach unten gerichtet. Das Foramen obturatorium erscheint beim Schafe kürzer und rundlicher als bei der Ziege.

Am Kopfe beschreibt die Profillinie beim Schafe in der Regel einen konvexen, bei der Ziege einen konkaven Bogen. Die beiden Hornfortsätze sind bei ersterem Thiere durch einen längeren und rundlichen, bei letzterem durch einen kürzeren und spitzwinkeligen $Z$ wischenraum von einander getrennt. Die Hornfortsätze selbst erscheinen bei der Ziege seitlich flach gedrückt, beim Schafe dagegen mehr rund. Die dorsale Fläche des Kopfes ist beim Schafe je nach den Rassen mehr 
oder weniger stark, bei der Ziege jedoch nur in geringem Grade gewölbt. Die beim Schafe unterhalb der Augenhöhle vorhandene Thränengrube fehlt bei der Ziege.

Das Schulterblatt des Schafes ist breiter und kürzer als das der Ziege; die Gräte des Schulterblattes ist beim Schafe stärker entwickelt und zeigt in der Mitte eine wulstartige Verdickung, welche im Bogen etwas nach hinten geworfen ist. Bei der Ziege hat die Gräte einen mehr flachen, durchaus geraden Verlauf; zudem ist dieselbe bedeutend niedriger als beim Schafe. Das Collum scapulae ist bei der Ziege deutlicher ausgeprägt.

Der Humerus ist bei der Ziege etwas länger, der Metacarpus dagegen kürzer als beim Schafe; auch ist das Armbein der Ziege schlanker, die Gelenk- und Muskelfortsätze an den beiden Endstücken dieses Knochens sind zierlicher und schwächer ausgebildet als beim Schafe. Bei ungefähr gleicher Länge des Vorarms und des Ellenbogenbeins bei beiden Thieren sind diese Knochen bei der Ziege schlanker und dünner als beim Schafe. Bei beiden Thieren findet sich an der lateralen Seite des Metacarpus ein Griffelbein.

Femur und Tibia sind bei der Ziege verhältnissmässig kürzer als beim Schafe; das Oberschenkelbein ist ferner erheblich schlanker und dünner, sowie der Hals des Gelenkkopfes viel schärfer ausgeprägt als beim Schafe, wodurch der Gelenkkopf der Ziege stärker hervortritt. Sämmtliche Fortsätze des Oberschenkelbeins sind feiner, die Kämme der Rollen stehen nicht so weit auseinander wie beim Schafe. Das Unterschenkelbein verläuft bei der Ziege nahezu gerade, beim Schafe erscheint die hintere Fläche dieses Knochens konkav. Zudem ist die Tibia des Schafes besonders am Kamme stärker spiralig gedreht als die der Ziege. Der Metatarsus der Ziege ist relativ kürzer als beim Schafe; er ist schlank und auf dem Querschnitte rundlich, beim Schafe dagegen breit und auf dem Querschnitte länglich.

Betrachten wir schliesslich die osteologischen Unterschiede zwischen Schaf und Ziege mit Rücksicht auf die Lebensweise und Bewegungsart beider Thiere, so dürfte die Behauptung gerechtfertigt erscheinen, dass die Lebensweise auf die Form des Skelets von wesentlichem Einfluss gewesen ist und die Unterschiede in dem Knochenbau dieser sonst so verwandten Thierarten allmählich hervorgebracht hat. Wenn auch Rütimeyer mit Recht die Urgeschichte der Schafe und Ziegen für eine der auffälligsten Lücken in der Palaeontologie der Säugethiere hält, so darf es nach Wilckens (13) doch als ausgemacht gelten, dass die gemeinsame Stammform von Schaf und Ziege durch die Familie der Antilopen führt. Während sich das Schaf weiter von seiner Stammform entfernt hat und nach Wilckens (13) nur in dem auf dem Hochlande von Tibet lebenden Takin (Budorcas Taxicola) eine vermittelnde oder Uebergangsform besitzt, fällt die Verwandtschaft zwischen Antilope und Ziege mehr in die Augen; zumal die zu den Antilopen gehörige Gruppe der Gemsen steht der Ziege ziemlich nahe. Die Ziege hat trotz der Domestikation ihr ursprüngliches Naturell weit mehr beibehalten als das Schaf: sie gehört dem Gebirgslande an und ist durch den Bau ihres 
Skelets für ihre Lebensweise und Bewegungsart ganz besonders befähigt. Die einzelnen Knochen der Wirbelsäule und namentlich die der Extremitäten zeichnen sich durch ihre schlanke Form aus und sind durch feinere Gelenke leicht beweglich mit einander verbunden. Die Röhrenknochen erscheinen durch ihre lange, schlanke Form zu einer ausgiebigeren Hebelwirkung geeignet, wodurch dem Thiere eine rasche Lokomotion ermöglicht wird: auch die übrigen Knochen der Ziege sind für eine behende und kletternde Bewegungsart im hohen Grade befähigt. Die Ziegen sind weit mehr als die Schafe reine Gebirgsthiere: am liebsten halten sie sich an klippenreichen Stellen der Hochgebirge auf. Nur im flachen Lande werden die Ziegen einzeln gehalten, in Gebirgsgegenden aber trifft man auch die Hausziegen, den wilden Ziegen ähnlich, heerdenweise an. Ihr Temperament ist ganz im Gegensatz zu den Schafen lebhaft und munter. Die Nahrung der Ziegen besteht vorwiegend in Gebirgspflanzen und trockenem Futter: hinsichtlich der Pflanzenarten sind sie sehr wählerisch, sodass die Art und Weise der Nahrungsaufnahme in der Regel mehr ein Naschen ist als ein wirkliches Befriedigen des Hungers.

Wenige Thiere haben durch ihre Kultur soviel von ihren ursprünglichen Eigenschaften verloren als das Schaf. Namentlich ist die dem Wildschafe gleichwie der Ziege eigenthümliche leichte Bewegungsart bei dem zahmen Schafe fast gänzlich verschwunden; die Bewegungen der Schafe sind langsam, ihr Lauf ist kurz und gar nicht anhaltend. Diese Eigenschaften prägen sich auch in dem Skelet des Schafes aus: Alle Knochen sind kürzer, gedrungener und massiger, die Gelenkverbindungen verhältnissmässig plumper als bei der Ziege. Die Bewegungsart des Schafes dürfte auch als ein Grund für die grosse Neigung zum Fettansatz anzusprechen sein, wodurch sich einige Schafrassen z. B. das Fettsteissschaf und das Fettschwanzschaf in ganz abnormer Weise auszeichnen. 


\section{Nachtrag.}

Das zoologische Museum der Universität Leipzig besitzt mehrere Skelete von Wildschafen (Ovis musimon Schreber), eines von der AngoraZiege (Capra hircus angorensis), sowie eines von der Gemse (Antilope rupicapra Erxleben), deren vergleichende Betrachtung eine nicht unwesentliche Ergänzung zu dem vorliegenden Thema bieten dürfte. Die Benutzung dieser Skelete wurde mir von dem Direktor des zoologischen Instituts, Herrn Geheimrath Prof Dr. Leuckart, in bereitwilligster Weise gestattet.

Die Skelete vom Mufflon zeigen uns die breite Stirn, sowie die konvexe Profillinie, welche dem Kopfe des Schafes eigenthümlich ist. Sämmtliche Halswirbel mit Ausnahme des Atlas weisen starke Dornfortsätze auf, die jedoch oben stumpf, niemals spitz sind wie bei der Ziege; der Kamm des Epistropheus hat ebenfalls eine mächtige Entwicklung. Die Dornfortsätze der Rücken- und Lendenwirbel sind etwas schlanker als beim zahmen Schaf, aber nichts destoweniger verhältnissmässig kräftig. Die Querfortsätze der Lendenwirbel sind auch hier etwas nach abwärts gerichtet. Das Becken ist dagegen etwas schmäler - auch an dem Skelet eines weiblichen Mufflon fällt dies sofort in die Augen - als beim zahmen Schaf, jedoch immerhin noch erheblich breiter als bei der Ziege. Das Schulterblatt sowie die Knochen der Extremitäten verhalten sich wie die gleichen Knochen des zahmen Schafes; nur erscheint beim Mufflon das Acromion scapulae deutlicher ausgeprägt als bei letzterem Thiere.

An dem Skelete der in Klein-Asien unweit des alten Ancyra heimischen Angora-Ziege finden wir die Flügelfortsätze des I. und den Kamm des 2. Halswirbels in gleicher Weise wie bei unserer Hausziege sehr stark entwickelt. Die Dornfortsätze der übrigen Hals- und der Rückenwirbel sind hoch, schmal und verhältnissmässig spitz; der I2. Rückenwirbel ist auch hier der diaphragmatische. Das Becken erscheint schmal und eng, die Gräte des Schulterblatts hoch und scharf.

Gleichwie bei der Ziege, so fehlt auch bei der Gemse das Querfortsatzloch am Epistropheus; der Kamm dieses Wirbels ist hier ebenfalls sehr stark entwickelt und überragt den Wirbelkörper nach vorn ganz erheblich. Die Dornfortsätze sind an den Hals- und Rückenwirbeln 
noch dünner und spitzer als bei der Ziege. Die Extremitätenknochen der Gemse, zumal Humerus und Radius, Femur und Tibia, erscheinen bei weitem länger und noch beträchtlich schlanker als die der Ziege; alle Fortsätze dieser Knochen sind äusserst fein und zierlich.

$\begin{array}{clll}\text { Länge des Humerus: } & \mathrm{I} 7,5 \mathrm{~cm} \\ \text { Radius: } & \mathrm{I} 8,3 \mathrm{~cm} \\ \text { Femur: } & 20 & \mathrm{~cm} \\ \text { Tibia: } & 26 \mathrm{~cm}\end{array}$

Aus der Betrachtung dieser Skelete erhellt in gleicher Weise, dass sich die einzelnen Unterschiede an den Knochen einer jeden der untersuchten Thierarten in der Weise allmählich entwickelt haben, wie sich beide Thiere entsprechend ihrer verschiedenen Lebensweise und Bewegungsart allmählich von einander differenzirten. Daher sind die Merkmale, die uns an den Knochen der wilden Thierarten auffallen, auch bei weitem geringer als die bei den zahmen Schafen und Ziegen. Die Knochen der letzteren haben sich seit Jahrhunderten und Jahrtausenden der verschiedenen Bewegungsart dieser Thiere angepasst, wodurch die osteologischen Unterschiede schliesslich stabil und konstant geworden sind. Die vergleichende Osteologie bietet uns also die Möglichkeit, die wichtigsten Merkmale der Knochen beider Thiere in eine bestimmte Reihe zu bringen, welche allerdings auch Seitenzweige treiben kann; als solche dürfen wir auch wohl die zahlreichen Rasseneigenthümlichkeiten bezeichen. Die vergleichende Betrachtung der Skelete beider Thierspezies lehrt uns wiederum, dass in der Natur nichts unvermittelt geschieht, sondern dass sich alles nur ganz allmählich entwickelt, stets getreu dem bekannten Satze: „Natura non facit saltus.“ 


\section{Literatur.}

I. Gurlt, E. F., Handbuch der Anatomie der Hausthiere. IV. Auflage.

2. Müller, C. in: Handbuch der vergleichenden Anatomie der Haussäugethiere, bearbeitet und herausgegeben von Leisering und Müller, VI. Auflage, bezw. Ellenberger und Müller, VII. Auflage. Berlin I896.

3. Eranck, L., Handbuch der vergleichenden Anatomie der Hausthiere, mit besonderer Berücksichtigung des Pferdes. I. Theil, III. Auflage. Stuttgart I892.

4. Nathusius, H. von (Hundisburg), Vorträge über Viehzucht und Rassenkenntniss. II. Theil: Schafzucht. Berlin 1872.

5. Settegast, H., Seperatabdruck des Vorworts zur III. Auflage der Thierzucht. p. IX. id., die Thierzucht.

6. Rohde, Schafzucht.

7. Mentzel, Schafzucht.

8. Martin, P., Die Beurtheilung der Herkunft des Fleisches nach den vorhandenen Knochentheilen, in Prof. Dr. Ostertag's Zeitschrift für Fleisch- und MilchHygiene. I. Jahrgang. Berlin I89I, Heft 5, p. 69 u. f.

9. Gi e be 1, Säugethiere. I859. p. 274 .

Iо. Zittel, C. A., Handbuch der Palaeontologie. I. Abtheilung. Band IV. p. 424. München und Leipzig I89I-I893.

I I. Murie, Monographische Anatomie der Saiga-Antilope. Procedings zoolog. Soc. London i 870 . p. 474.

I 2. Bonnet, R., Grundriss der Entwickelungsgeschichte der Haussäugethiere. Berlin I89I. p. I 74--205.

I3. Wilckens, M., Grundriss der Naturgeschichte der Haussäugethiere. Dresden i 880 .

I4. Sussdorf, M., Lebrbuch der vergleichenden Anatomie der Hausthiere. Stuttgart 1895 . 


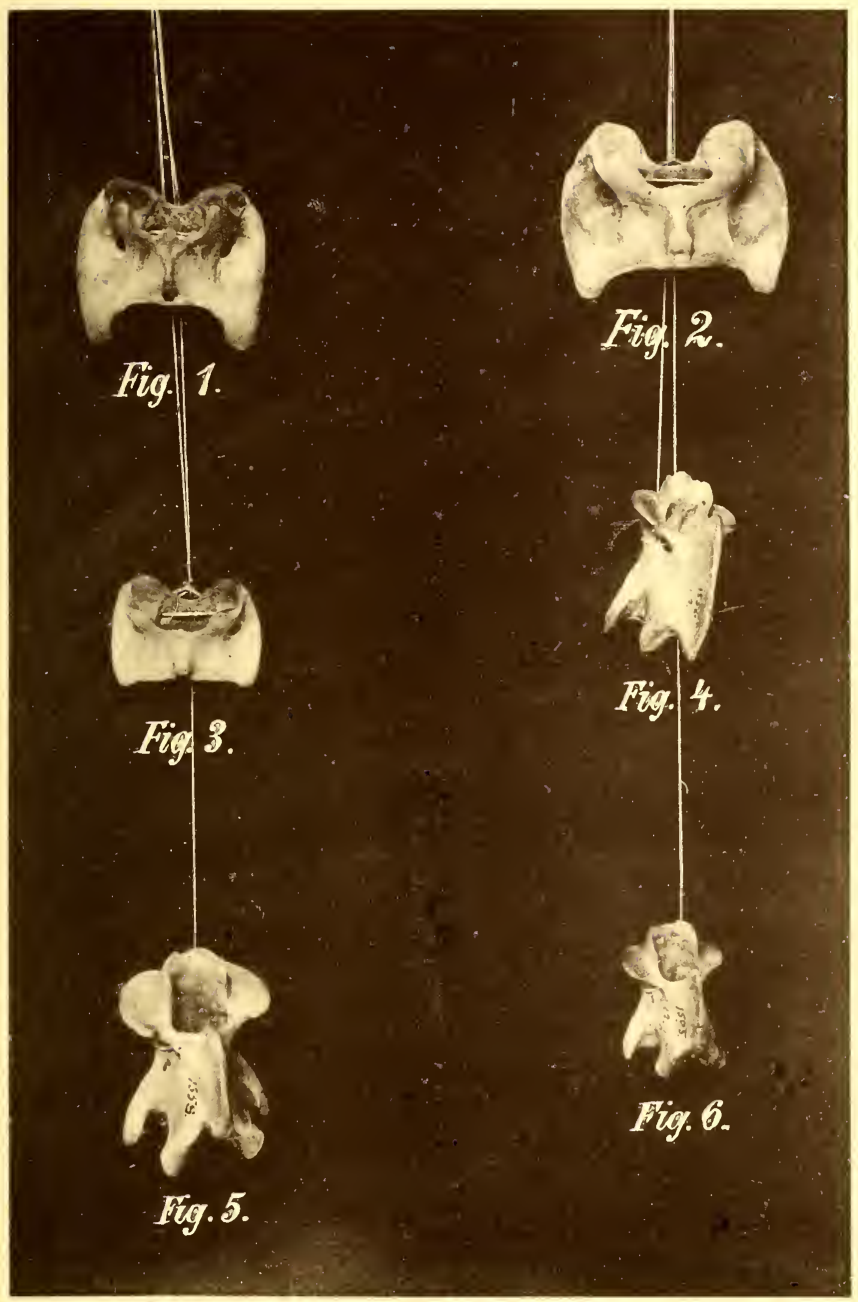

TAFEL I.

Fig. I.

Atlas der Ziege von unten.

Fig. 2.

Atlas des Marschschafes von unten.

Fig. 3 .

Atlas der Heidschnucke von unten.
Fig. 4 .

Epistropheus der Ziege von oben.

Fig. 5 .

Epistropheus des Marschschafes von oben.

Fig. 6.

Epistropheus der Heidschnucke von oben. 



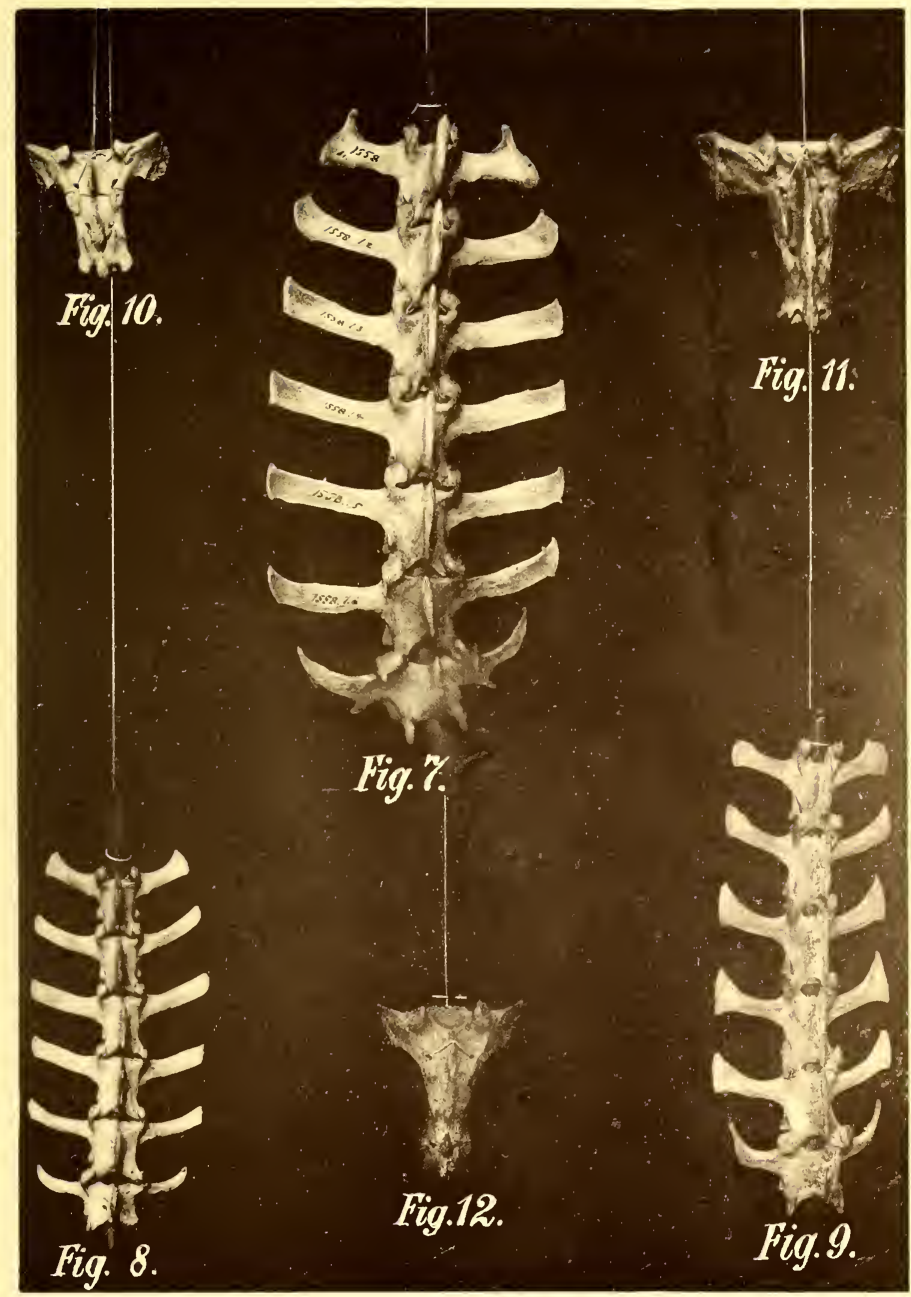

TAFEL II.

Fig. 7 .

Lendenwirbel des Marschschafes von oben.

Fig. 8.

Lendenwirbel der Heidschnucke von oben.

Fig. 9.

Lendenwirbel der Ziege von oben.
Fig. 10.

Kreuzbein der Heidschnucke von oben. Fig. II.

Kreuzbein des Marschschafes von oben. Fig. I 2.

Kreuzbein der Ziege von oben. 



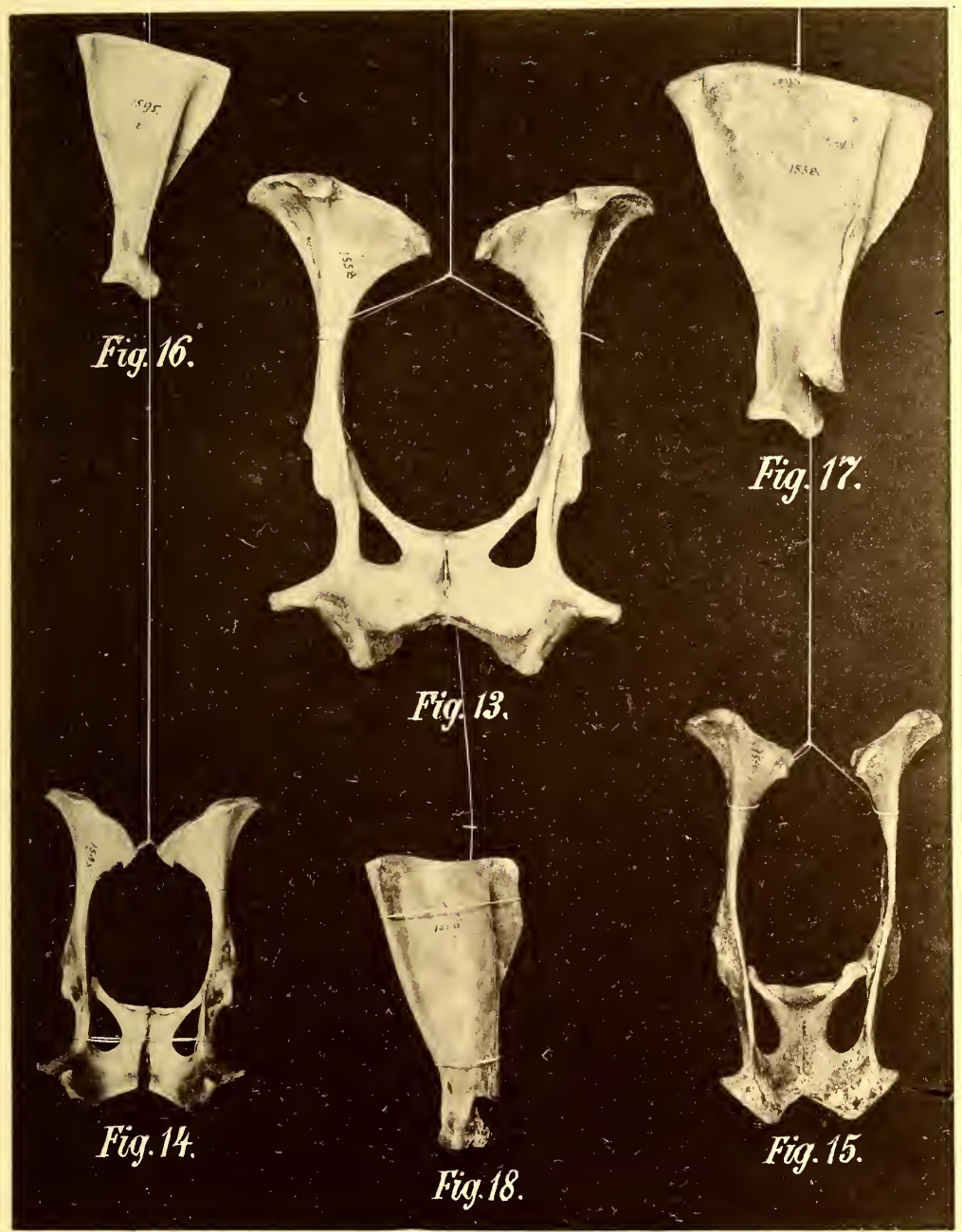

T A FEL III.

Fig. I3.

Becken des Marschschafes von oben.

Fig. I 4.

Becken der Heidschnucke von oben.

Fig. I 5 .

Becken der Ziege von oben.
Fig. I 6.

Schulterblatt der Heidschnucke.

Fig. 17 .

Schulterblatt des Marschschafes.

Fig. 18.

Schulterblatt der Ziege. 



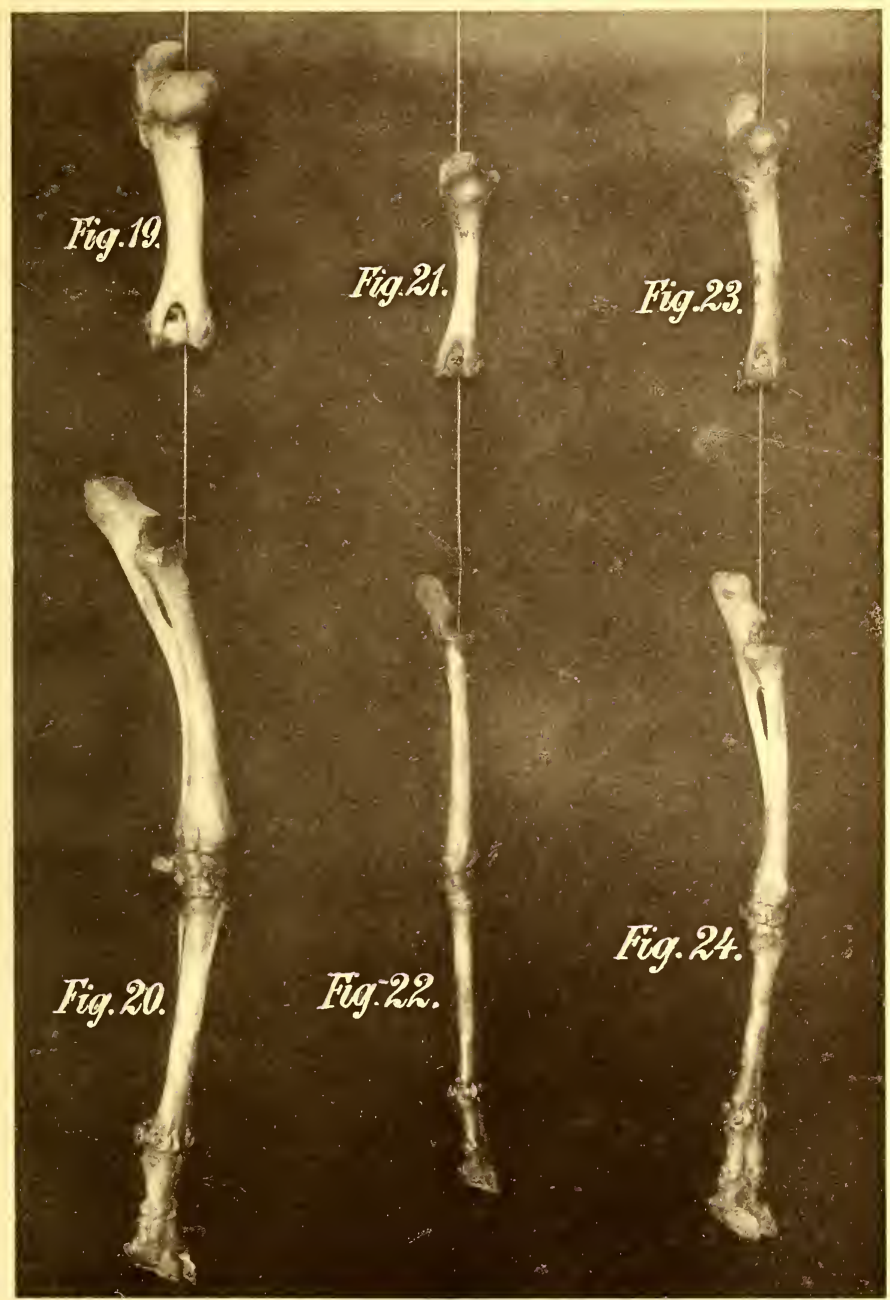

TAFEL IV.

Fig. 19.

Humerus des Marschschafes von vorn.

Fig. 20.

Rechte untere Vorder-Extremitäten knochen des Marschschafes, Seite.

Fig. 2 I.

Humerus der Heidschnucke von vorn.
Fig. 22.

Linke untere Vorder-Extremitätenknochen der Heidschnucke.

Fig. 23.

Humerus der Ziege von vorn.

Fig. 24.

Rechte untere Vorder-Extremitäten knochen der Ziege, Seite. 



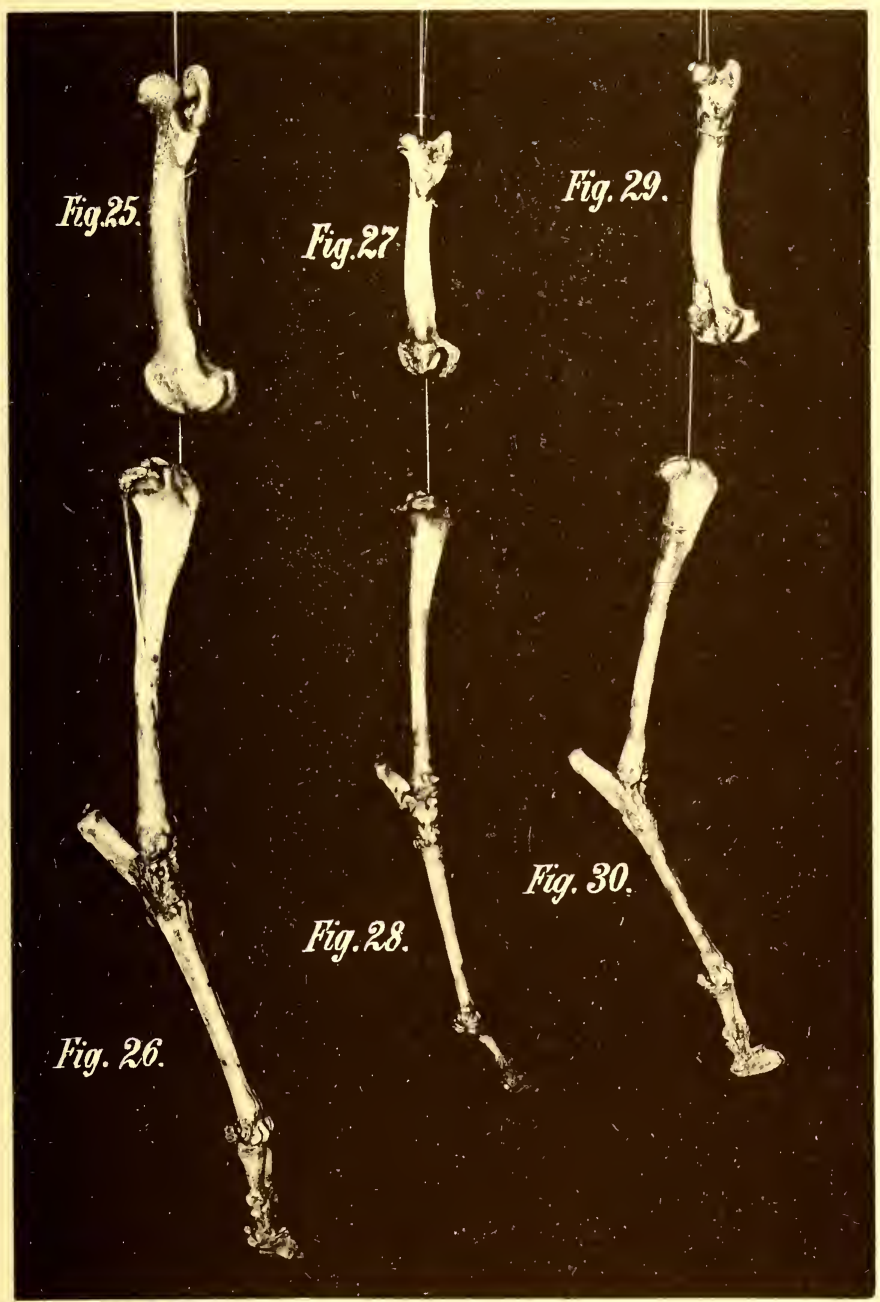

TAFEL V.

Fig. 25.

Femur des Marschschafes von der lateralen und vordern Seite.

Fig. 26.

Rechte untere Hinter-Extremitätenknochen des Marschschafes, Seite.

Fig. 27.

Femur der Heidschnucke von der lateralen und vordern Seite.
Fig. 28.

Linke untereHinter-Extremitätenknochen der Heidschnucke, Seite.

Fig. 29.

Femur der Ziege von der lateralen und vordern Seite.

Fig. 30.

Rechte untere Hinter-Extremitätenknochen der Ziege, Seite. 



\section{Currieulum vitae.}

Carl Hubert Bützler wurde geboren den I2. Juni I867 zu Bergisch-Gladbach im Kreise Mülheim am Rhein, Regierungsbezirk Köln, und ist der dritte Sohn des im Jahre I888 zu Kalk, Landkreis Köln, verstorbenen Kaufmannes Heinrich Bützler. Er wurde in der katholischen Religion erzogen und besuchte vom 6. bis zum I4. Lebensjahre die Elementarschule in Kalk. Nachdem er durch Privatunterricht in den Gymnasialfächern vorgebildet worden war, konnte er zum Ostertermin I883 nach Ablegung der Aufnahmeprüfung am Königlichen Gymnasium an der Apostelnkirche zu Köln in die Obertertia aufgenommen werden. Dieses Gymnasium besuchte er bis Ostern 1888, wo er das Zeugniss für die Oberprima erhielt, und begann darauf an der Königlichen thierärztlichen Hochschule zu Hannover das Studium der Thierheilkunde. In seinem 4. Studiensemester unterzog er sich mit gutem Erfolg der naturwissenschaftlichen Prüfung an dieser Lehranstalt und besuchte nach Ablauf des Sommer-Semesters 1890 die Grossherzoglich Hessische Veteriväranstalt der Universität Giessen, wo sich ihm Gelegenheit bot, neben den Studium der Veterinär-Medizin das der Fächer Zoologie, Botanik und Physik weiter zu betreiben. Mit Beginn seines 6. Semesters kehrte er nach Hannover zur thierärztlichen Hochschule zurück, blieb hier die beiden letzten Semester hindurch und bestand Ostern I 892 vor der Königlich Preussischen Prüfungs-Kommission die thierärztliche Fachprüfung mit dem Prädikate "gut", worauf ihm der thierärztliche Approbationsschein ertheilt wurde.

Bis zum Frühjahre 1893 prakticirte er in Kirn an der Nahe und wurde alsdann zum Vorsteher des städtischen Schlachthauses zu Jülich ernannt. Seit dem I. Juni I 894 ist der Unterzeichnete mit der Leitung des städtischen Schlachthofes zu Trier betraut und bekleidet diese Stelle, für welche er auf Lebenszeit angestellt worden ist, zur vollen Zufriedenheit seiner vorgesetzten Behörde.

Das Studium der Naturwissenschaften betrieb der Unterzeichnete seit der Erlangung der Approbation als Thierarzt in seiner freien Zeit privatim weiter und hörte im Winter-Semester $1895 / 96$ an der Universität Bonn Vorlesungen über Zoologie, Botanik und Physik, worauf er zur Beendigung seiner als Inaugural-Dissertation bestimmten Arbeit: "Zur vergleichenden Osteologie des Schafes und der Ziege" vor Beginn des verflossenen Sommer-Semesters nach Berlin ging, um hier gleichzeitig am zoologischen Institut der Universität, an der landwirthschaftlichen, sowie an der thierärztlichen Hochschule das Untersuchungs-Material für seine Arbeit benutzen zu können. Am r. Juni d. J. ist der Unterzeichnete an der Universität Leipzig für zwei Semester immatrikulirt worden.

Leipzig, den 28. Oktober I 896.

\section{Bützler,}

approb. Thierarzt und Schlachthof-Direktor. 
SMITHSONIAN INSTITUTION LIBRARIES

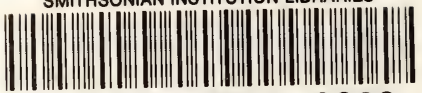

39088007199920 